\title{
On Witt vector cohomology for singular varieties
}

\author{
Pierre Berthelot, Spencer Bloch and Hélène Esnault
}

\begin{abstract}
Over a perfect field $k$ of characteristic $p>0$, we construct a 'Witt vector cohomology with compact supports' for separated $k$-schemes of finite type, extending (after tensorization with $\mathbb{Q}$ ) the classical theory for proper $k$-schemes. We define a canonical morphism from rigid cohomology with compact supports to Witt vector cohomology with compact supports, and we prove that it provides an identification between the latter and the slope $<1$ part of the former. Over a finite field, this allows one to compute congruences for the number of rational points in special examples. In particular, the congruence modulo the cardinality of the finite field of the number of rational points of a theta divisor on an abelian variety does not depend on the choice of the theta divisor. This answers positively a question by J.-P. Serre.
\end{abstract}

\section{Introduction}

Let $k$ be a perfect field of characteristic $p>0, W=W(k), K=\operatorname{Frac}(W)$. If $X$ is a proper and smooth variety defined over $k$, the theory of the de Rham-Witt complex and the degeneration of the slope spectral sequence provide a functorial isomorphism (see [Blo77, III, Example 3.5] and [Ill79, II, Corollaire 3.5])

$$
H_{\text {crys }}^{*}(X / K)^{<1} \stackrel{\sim}{\longrightarrow} H^{*}\left(X, W \mathcal{O}_{X}\right)_{K},
$$

where $H_{\text {crys }}^{*}(X / K)^{<1}$ is the maximal subspace on which Frobenius acts with slopes $<1$, and the subscript $K$ denotes tensorization with $K$.

If we only assume that $X$ is proper, but may be singular, we can use rigid cohomology to generalize crystalline cohomology while retaining all of the standard properties of a topological cohomology theory. Thus, the left-hand side of (1.1) remains well defined and, when $k$ is finite, the alternated product of the corresponding characteristic polynomials of Frobenius can be interpreted as the factor of the zeta function $\zeta(X, t)$ 'of slopes $<1$ ' (see $\S 6.1$ for a precise definition). On the other hand, the classical theory of the de Rham-Witt complex can no longer be directly applied to $X$, but the sheaf of Witt vectors $W \mathcal{O}_{X}$ is still available. Thus, the right-hand side of (1.1) also remains well defined. As in the smooth case, this is a finitely generated $K$-vector space endowed with a Frobenius action with slopes in $[0,1$ [ (Proposition 2.10), which has the advantage of being directly related to the coherent cohomology of $X$. It is therefore of interest to know whether, when $X$ is singular, (1.1) can be generalized as an isomorphism

$$
H_{\text {rig }}^{*}(X / K)^{<1} \stackrel{\sim}{\longrightarrow} H^{*}\left(X, W \mathcal{O}_{X}\right)_{K},
$$

where $H_{\text {rig }}^{*}(X / K)^{<1}$ denotes the subspace of slope $<1$ of $H_{\text {rig }}^{*}(X / K)$.

Our main result gives a positive answer to this question. More generally, we show that a 'Witt vector cohomology with compact supports' can be defined for separated $k$-schemes of finite type,

Received 18 October 2005, accepted in final form 16 June 2006.

2000 Mathematics Subject Classification 11G25, 14F30, 14G05, $14 \mathrm{G} 10$.

Keywords: Witt vectors, rigid cohomology, slope filtration, zeta function, rational points.

The first author has been supported by the research network Arithmetic Algebraic Geometry of the European Community (Contract MRTN-CT-2003-504917). The third author has been supported by the DFG-Leibniz program. This journal is (C) Foundation Compositio Mathematica 2007. 


\section{P. Berthelot, S. Bloch and H. Esnault}

giving cohomology spaces $H_{\mathrm{c}}^{*}\left(X, W \mathcal{O}_{X, K}\right)$ which are finite-dimensional $K$-vector spaces, endowed with a Frobenius action with slopes in $[0,1[$. Then, for any such scheme $X$, the slope $<1$ subspace of the rigid cohomology with compact supports of $X$ has the following description.

TheORem 1.1. Let $k$ be a perfect field of characteristic $p>0$ and let $X$ be a separated $k$-scheme of finite type. There exists a functorial isomorphism

$$
H_{\text {rig,c }}^{*}(X / K)^{<1} \stackrel{\sim}{\longrightarrow} H_{\mathrm{c}}^{*}\left(X, W \mathcal{O}_{X, K}\right) .
$$

This is a striking confirmation of Serre's intuition [Ser58a] about the relation between topological and Witt vector cohomologies. On the other hand, this result bears some analogy with Hodge theory. Recall from [BH69] (or [Del74]) that if $X$ is a proper scheme defined over $\mathbb{C}$, then its Betti cohomology $H^{*}(X, \mathbb{C})$ is a direct summand of its de Rham cohomology $\mathbb{H}^{*}\left(X, \Omega_{X}^{\bullet}\right)$. Coherent cohomology $H^{*}\left(X, \mathcal{O}_{X}\right)$ does not exactly compute the corner piece of the Hodge filtration, as would be an exact analogy with the formulation of Theorem 1.1, but it gives an upper bound. Indeed, by [Esn90, Proposition 1.2] and [Esn05, Proof of Theorem 1.1], one has a functorial surjective map $H^{*}\left(X, \mathcal{O}_{X}\right) \rightarrow \operatorname{gr}_{F}^{0} H^{*}(X, \mathbb{C})$.

The construction of these Witt vector cohomology spaces is given in $\S 2$. If $U$ is a separated $k$-scheme of finite type, $U \hookrightarrow X$ an open immersion in a proper $k$-scheme, and $\mathcal{I} \subset \mathcal{O}_{X}$ any coherent ideal such that $V(\mathcal{I})=X \backslash U$, we define $W \mathcal{I}=\operatorname{Ker}\left(W \mathcal{O}_{X} \rightarrow W\left(\mathcal{O}_{X} / \mathcal{I}\right)\right)$, and we show that the cohomology spaces $H^{*}\left(X, W \mathcal{I}_{K}\right)$ actually depend only on $U$ (Theorem 2.4). This results from an extension to Witt vectors of Deligne's results on the independence on the compactification for the construction of the $f$ ! functor for coherent sheaves [Har66].

When $U$ varies, these spaces are contravariant functors with respect to proper maps, and covariant functors with respect to open immersions. In particular, they give rise to the usual long exact sequence relating the cohomologies of $U$, of an open subset $V \subset U$, and of the complement $T$ of $V$ in $U$. Thus, they can be viewed as providing a notion of Witt vector cohomology spaces with compact support for $U$. We define

$$
H_{\mathrm{c}}^{*}\left(U, W \mathcal{O}_{U, K}\right):=H^{*}\left(X, W \mathcal{I}_{K}\right) .
$$

Among other properties, we prove in $\S 3$ that these cohomology spaces satisfy a particular case of cohomological descent (Theorem 3.2) which will be used in the proof of Theorem 1.1.

The construction of the canonical homomorphism between rigid and Witt vector cohomologies is given in $\S 4$ (Theorem 4.5). First, we recall how to compute rigid cohomology for a proper $k$-scheme $X$ when there exists a closed immersion of $X$ in a smooth formal $W$-scheme $\mathbb{P}$, using the de Rham complex of $\mathbb{P}$ with coefficients in an appropriate sheaf of $\mathcal{O}_{\mathbb{P}}$-algebras $\mathcal{A}_{X, \mathbb{P}}$. When $\mathbb{P}$ can be endowed with a lifting of the absolute Frobenius endomorphism of its special fibre, a simple construction (based on an idea of Illusie [Ill79]) provides a functorial morphism from this de Rham complex to $W \mathcal{O}_{X, K}$. Using Cech resolutions, this morphism can still be defined in the general case as a morphism in the derived category of sheaves of $K$-vector spaces. Then we obtain (1.3) by taking the morphism induced on cohomology and restricting to the slope $<1$ subspace. By means of simplicial resolutions based on de Jong's theorem, we prove in $\S 5$ that it is an isomorphism. The proof proceeds by reduction to the case of proper and smooth schemes, using Theorem 3.2 and the descent properties of rigid cohomology proved by Chiarellotto and Tsuzuki [CT03, Tsu03, Tsu04].

We now list some applications of Theorem 1.1, which are developed in $\S 6$. We first remark that it implies a vanishing theorem. If $X$ is smooth projective, and $Y \subset X$ is a divisor so that $U=X \backslash Y$ is affine, then Serre vanishing says that $H^{i}\left(X, \mathcal{O}_{X}(-n Y)\right)=0$ for $n$ large and $i<\operatorname{dim}(X)$. If $k$ has characteristic 0 , then we can take $n=1$ by Kodaira vanishing. One then has $H^{i}\left(X, \mathcal{O}_{X}(-Y)\right)=0$ for $i<\operatorname{dim}(X)$. It is tempting to view the following corollary as an analogue when $k$ has characteristic $p$. 


\section{WitT VECTOR COHOMOLOGY OF SINGULAR VARIETIES}

COROllary 1.2. Let $X$ be a proper scheme defined over a perfect field $k$ of characteristic $p>0$. Let $\mathcal{I} \subset \mathcal{O}_{X}$ be a coherent ideal defining a closed subscheme $Y \subset X$ such that $U=X \backslash Y$ is affine, smooth and equidimensional of dimension $n$. Then

$$
H_{c}^{i}\left(U, W \mathcal{O}_{U, K}\right)=H^{i}(X, W \mathcal{I})_{K}=H^{i}\left(X, W \mathcal{I}^{r}\right)_{K}=0
$$

for all $i \neq n$ and all $r \geqslant 1$.

Indeed, when $U$ is smooth and affine, $H_{\text {rig }}^{i}(U / K)$ can be identified with Monsky-Washnitzer cohomology [Ber97a], and therefore vanishes for $i>n=\operatorname{dim}(U)$. If, moreover, $U$ is equidimensional, it follows by Poincaré duality [Ber97b] that $H_{\text {rig,c }}^{i}(U / K)=0$ for $i<n$. So (1.3) implies that $H^{i}(X, W \mathcal{I})_{K}=0$ for $i<n$. On the other hand, the closure $\bar{U}$ of $U$ in $X$ has dimension $n$, and $H^{i}(X, W \mathcal{I})_{K}=H_{\mathrm{c}}^{i}\left(U, W \mathcal{O}_{U, K}\right)$ does not change up to canonical isomorphism if we replace $X$ by $\bar{U}$. Therefore, $H^{i}(X, W \mathcal{I})_{K}=0$ for $i>n$.

When $k$ is a finite field $\mathbb{F}_{q}$, with $q=p^{a}$, Theorem 1.1 implies a statement about zeta functions. By [EL93, Théorème II], the Lefschetz trace formula provides an expression of the $\zeta$-function of $X$ as the alternating product

$$
\zeta(X, t)=\prod_{i} P_{i}(X, t)^{(-1)^{i+1}}
$$

where $P_{i}(X, t)=\operatorname{det}\left(1-t \phi \mid H_{\text {rig, },}^{i}(X / K)\right)$, and $\phi=F^{a}$ denotes the $\mathbb{F}_{q}$-linear Frobenius endomorphism of $X$. On the other hand, we define

$$
\begin{aligned}
P_{i}^{W}(X, t) & =\operatorname{det}\left(1-t \phi \mid H_{\mathrm{c}}^{i}\left(X, W \mathcal{O}_{X, K}\right)\right), \\
\zeta^{W}(X, t) & =\prod_{i} P_{i}^{W}(X, t)^{(-1)^{i+1}} .
\end{aligned}
$$

If we denote by $\zeta^{<1}(X, t)$ the 'slope $<1$ factor' of $\zeta(X, t)$ (cf. $\S 6.1$ ), we formally obtain the following from (1.3).

Corollary 1.3. Let $X$ be a separated scheme of finite type over a finite field. Then one has

$$
\zeta^{<1}(X, t)=\zeta^{W}(X, t)
$$

This result can be used to prove congruences $\bmod q$ on the number of $\mathbb{F}_{q}$-rational points of certain varieties. The following theorem answers a question of Serre, and was the initial motivation for this work. Recall that an effective divisor $D$ on an abelian variety $A$ is called a theta divisor if $\mathcal{O}_{A}(D)$ is ample and defines a principal polarization.

Theorem 1.4. Let $\Theta, \Theta^{\prime}$ be two theta divisors on an abelian variety defined over a finite field $\mathbb{F}_{q}$. Then

$$
\left|\Theta\left(\mathbb{F}_{q}\right)\right| \equiv\left|\Theta^{\prime}\left(\mathbb{F}_{q}\right)\right| \bmod q
$$

Actually, Serre's original formulation predicts that, on an abelian variety defined over a field, the difference of the motives of $\Theta$ and $\Theta^{\prime}$ is divisible by the Lefschetz motive. Our Theorem 1.4 answers the point counting consequence of it.

We also have more elementary point counting consequences.

Corollary 1.5 (Ax [Ax64], Katz [Kat71]). Let $D_{1}, \ldots, D_{r} \subset \mathbb{P}^{n}$ be hypersurfaces of degrees $d_{1}, \ldots, d_{r}$, defined over the finite field $\mathbb{F}_{q}$. Assume that $\sum_{j} d_{j} \leqslant n$. Then

$$
\left|\left(D_{1} \cap \cdots \cap D_{r}\right)\left(\mathbb{F}_{q}\right)\right| \equiv 1 \bmod q .
$$

We observe here that this congruence is the best approximation of the results of Ax and Katz that can be obtained using Witt vector cohomology, since this method only provides information 


\section{P. Berthelot, S. Bloch and H. Esnault}

on the slope $<1$ factor of the zeta function. It would be worthwhile to have results similar to Theorem 1.1 for higher slopes which might give the full Ax-Katz congruences.

We also remark that Ax's theorem has a motivic proof [BEL05], which of course is more powerful than this slope proof. Yet it is of interest to remark that Theorem 1.1 applies here as well. As for Theorem 1.4, it seems more difficult to formulate a motivic proof, as it would have to deal with non-effective motives (see the discussion in Remarks 6.6).

Let us mention finally the following general consequence of Theorem 1.1, which for example can be applied in the context of the work of $\mathrm{Fu}$ and Wan on mirror congruences for Calabi-Yau varieties [Wan04, FW05].

Corollary 1.6. Let $f: X \rightarrow Y$ be a morphism between two proper $\mathbb{F}_{q^{-s c h e m e s}}$. If $f$ induces isomorphisms $f^{*}: H^{i}\left(Y, \mathcal{O}_{Y}\right) \stackrel{\sim}{\longrightarrow} H^{i}\left(X, \mathcal{O}_{X}\right)$ for all $i \geqslant 0$, then

$$
\left|X\left(\mathbb{F}_{q}\right)\right| \equiv\left|Y\left(\mathbb{F}_{q}\right)\right| \bmod q .
$$

\section{Notation and conventions}

Throughout this article, $k$ denotes a perfect field of characteristic $p, W=W(k), \sigma: W \rightarrow W$ is the Frobenius automorphism of $W, K=\operatorname{Frac}(W)$. The subscript $K$ will denote tensorization with $K$ over $W$. We recall that, on any noetherian topological space, taking cohomology commutes with tensorization by $\mathbb{Q}$. Therefore, the subscript ${ }_{K}$ will be moved inside or outside cohomology or direct images without further justification.

We denote by $D^{\mathrm{b}}(K)$ (respectively $D^{\mathrm{b}}(X, K)$ ) the derived category of bounded complexes of $K$-vector spaces (respectively complexes of sheaves of $K$-vector spaces on a topological space $X$ ).

All formal schemes considered in this article are $W$-formal schemes for the $p$-adic topology.

\section{Witt vector cohomology with compact supports}

We give in this section some properties of Witt vector cohomology which are a strong indication of its topological nature, and will be used later in our proof of Theorem 1.1. In particular, we show how to attach Witt vector cohomology spaces with compact supports to any separated $k$-scheme of finite type.

If $X$ is a scheme, $\mathcal{A}$ a sheaf of rings on $X$, and $n \geqslant 1$, we denote by $W_{n} \mathcal{A}$, or by $W_{n}(\mathcal{A})$ if confusion may arise, the sheaf of Witt vectors of length $n$ with coefficients in $\mathcal{A}$, and by $W \mathcal{A}=$ $\lim _{n} W_{n} \mathcal{A}$, or $W(\mathcal{A})$, the sheaf of Witt vectors of infinite length. If $\mathcal{I} \subset \mathcal{A}$ is an ideal, we denote by $\overleftarrow{W}_{n}^{n} \mathcal{I}=\operatorname{Ker}\left(W_{n} \mathcal{A} \rightarrow W_{n}(\mathcal{A} / \mathcal{I})\right)$, or $W_{n}(\mathcal{I})$, the sheaf of Witt vectors $\left(a_{0}, a_{1}, \ldots, a_{n-1}\right)$ such that $a_{i}$ is a section of $\mathcal{I}$ for all $i$, and we define similarly $W \mathcal{I}$. Note that, when $\mathcal{I}$ is quasi-coherent, the canonical morphism $W \mathcal{I} \rightarrow \mathbb{R} \lim _{n} W_{n} \mathcal{I}$ is an isomorphism, as $H^{1}\left(U, W_{n} \mathcal{I}\right)=0$ for any affine open subset $U \subset X$ and any $n$, and the projective system $\Gamma\left(U, W_{n} \mathcal{I}\right)$ has surjective transition maps.

For any $X$, any sheaf of rings $\mathcal{A}$ on $X$, and any ideal $\mathcal{I} \subset \mathcal{A}$, we use the notation $\mathbb{R} \Gamma(X, W \mathcal{I})$ and $H^{*}(X, W \mathcal{I})$ to denote the Zariski cohomology of the sheaf $W \mathcal{I}$. Thus, when $\mathcal{I}$ is quasi-coherent, the canonical morphism

$$
\mathbb{R} \Gamma(X, W \mathcal{I}) \rightarrow \underset{n}{\mathbb{R}} \lim _{n} \mathbb{R} \Gamma\left(X, W_{n} \mathcal{I}\right)
$$

is an isomorphism. When $X$ is a proper $k$-scheme and $\mathcal{I} \subset \mathcal{O}_{X}$ is a coherent ideal, the cohomology modules $H^{*}\left(X, W_{n} \mathcal{I}\right)$ are artinian $W$-modules; then it follows from the Mittag-Leffler criterion that the morphism

$$
H^{*}(X, W \mathcal{I}) \rightarrow \underset{n}{\lim _{n}} H^{*}\left(X, W_{n} \mathcal{I}\right)
$$

is an isomorphism. 


\section{WiTT VECTOR COHOMOLOGY OF SINGULAR VARIETIES}

We shorten notation by writing $W \mathcal{O}_{X, K}, W \mathcal{I}_{K}$ for $\left(W \mathcal{O}_{X}\right)_{K},(W \mathcal{I})_{K}$. We recall again that, when $X$ is noetherian, there is a canonical isomorphism

$$
\mathbb{R} \Gamma(X, W \mathcal{I})_{K} \stackrel{\sim}{\longrightarrow} \Gamma\left(X, W \mathcal{I}_{K}\right) .
$$

In this article, we are particularly interested in the $K$-vector spaces $H^{*}\left(X, W \mathcal{O}_{X, K}\right)$, and in their generalizations $H^{*}\left(X, W \mathcal{I}_{K}\right)$. Our main observation is that, in contrast to the $W$-modules $H^{*}\left(X, W \mathcal{O}_{X}\right)$, which are sensitive to nilpotent sections of $\mathcal{O}_{X}$, they behave like a topological cohomology theory for separated $k$-schemes of finite type. The following easy proposition is somehow a key point.

Proposition 2.1. Let $X$ be a $k$-scheme of finite type.

(i) The canonical homomorphism

$$
W \mathcal{O}_{X, K} \rightarrow W \mathcal{O}_{X_{\text {red }}, K}
$$

is an isomorphism, and induces a functorial isomorphism

$$
H^{*}\left(X, W \mathcal{O}_{X, K}\right) \stackrel{\sim}{\longrightarrow} H^{*}\left(X_{\mathrm{red}}, W \mathcal{O}_{X_{\mathrm{red}}, K}\right),
$$

compatible with the action of $F$ and $V$.

(ii) Let $\mathcal{I}, \mathcal{J} \subset \mathcal{O}_{X}$ be coherent ideals, and assume $\sqrt{\mathcal{I}}=\sqrt{\mathcal{J}}$, i.e. there exists $N \geqslant 1$ such that $\mathcal{I}^{N} \subset \mathcal{J}$ and $\mathcal{J}^{N} \subset \mathcal{I}$. Then there is a canonical identification

$$
W \mathcal{I}_{K} \cong W \mathcal{J}_{K}
$$

inducing a functorial isomorphism

$$
H^{*}\left(X, W \mathcal{I}_{K}\right) \cong H^{*}\left(X, W \mathcal{J}_{K}\right),
$$

compatible with the action of $F$ and $V$.

Proof. Let $\mathcal{N}=\operatorname{Ker}\left(\mathcal{O}_{X} \rightarrow \mathcal{O}_{X_{\text {red }}}\right)$. To prove the first claim of assertion (i), it suffices to show that $W \mathcal{N}_{K}=0$. However, the action of $p$ is invertible, and $p=V F=F V$, so it suffices to show that $F$ is nilpotent. This is clear since $F$ acts on $W \mathcal{N}$ by raising coordinates to the $p$ th power. Taking cohomology, the second claim follows.

Assertion (ii) follows from assertion (i) applied to $\mathcal{O}_{X} / \mathcal{I}$ and $\mathcal{O}_{X} / \mathcal{J}$.

We now prove the existence of Mayer-Vietoris exact sequences for Witt vector cohomology.

Proposition 2.2. Let $X$ be a $k$-scheme of finite type, $X_{1}, X_{2} \subset X$ two closed subschemes such that $X=X_{1} \cup X_{2}$ and $Z=X_{1} \cap X_{2}$. There is an exact sequence

$$
0 \rightarrow W \mathcal{O}_{X, K} \rightarrow W \mathcal{O}_{X_{1}, K} \oplus W \mathcal{O}_{X_{2}, K} \rightarrow W \mathcal{O}_{Z, K} \rightarrow 0
$$

providing a Mayer-Vietoris long exact sequence

$$
\begin{aligned}
\cdots & \rightarrow H^{i}\left(X, W \mathcal{O}_{X, K}\right) \rightarrow H^{i}\left(X_{1}, W \mathcal{O}_{X_{1}, K}\right) \oplus H^{i}\left(X_{2}, W \mathcal{O}_{X_{2}, K}\right) \\
& \rightarrow H^{i}\left(Z, W \mathcal{O}_{Z, K}\right) \rightarrow H^{i+1}\left(X, W \mathcal{O}_{X, K}\right) \rightarrow \cdots .
\end{aligned}
$$

Proof. Let $\mathcal{I}_{1}, \mathcal{I}_{2}$ be the ideals of $\mathcal{O}_{X}$ defining $X_{1}$ and $X_{2}$. Thanks to Proposition 2.1, we may assume that $\mathcal{O}_{Z}=\mathcal{O}_{X} /\left(\mathcal{I}_{1}+\mathcal{I}_{2}\right)$. It is easy to check that $W\left(\mathcal{I}_{1}+\mathcal{I}_{2}\right)=W\left(\mathcal{I}_{1}\right)+W\left(\mathcal{I}_{2}\right)$. From the exact sequence

$$
0 \rightarrow \mathcal{O}_{X} /\left(\mathcal{I}_{1} \cap \mathcal{I}_{2}\right) \rightarrow \mathcal{O}_{X} / \mathcal{I}_{1} \oplus \mathcal{O}_{X} / \mathcal{I}_{2} \rightarrow \mathcal{O}_{X} /\left(\mathcal{I}_{1}+\mathcal{I}_{2}\right) \rightarrow 0
$$

we can then deduce an exact sequence

$$
0 \rightarrow W\left(\mathcal{O}_{X} /\left(\mathcal{I}_{1} \cap \mathcal{I}_{2}\right)\right) \rightarrow W \mathcal{O}_{X_{1}} \oplus W \mathcal{O}_{X_{2}} \rightarrow W \mathcal{O}_{Z} \rightarrow 0
$$




\section{P. Berthelot, S. Bloch and H. Esnault}

Since $X=X_{1} \cup X_{2}, \mathcal{I}_{1} \cap \mathcal{I}_{2}$ is a nilpotent ideal, and Proposition 2.1 implies that $W \mathcal{O}_{X, K} \stackrel{\sim}{\longrightarrow}$ $W\left(\mathcal{O}_{X} /\left(\mathcal{I}_{1} \cap \mathcal{I}_{2}\right)\right)_{K}$. This gives the short exact sequence of the statement. The long exact sequence follows by taking cohomology.

Corollary 2.3. Let $X$ be a $k$-scheme of finite type, $X_{1}, \ldots, X_{r} \subset X$ closed subschemes such that $X=X_{1} \cup \cdots \cup X_{r}$. For each sequence $1 \leqslant i_{0}<\cdots<i_{n} \leqslant r$, let $X_{i_{0}, \ldots, i_{n}}=X_{i_{0}} \cap \cdots \cap X_{i_{n}}$. Then the sequence

$$
0 \rightarrow W \mathcal{O}_{X, K} \rightarrow \prod_{i=1}^{r} W \mathcal{O}_{X_{i}, K} \rightarrow \cdots \rightarrow W \mathcal{O}_{X_{1, \ldots, r}, K} \rightarrow 0
$$

is exact.

Proof. The statement being true for $r=2$, we proceed by induction on $r$. Let $X^{\prime}=X_{2} \cup \cdots \cup X_{r}$. Up to a shift, the complex (2.2) is the cone of the morphism of complexes

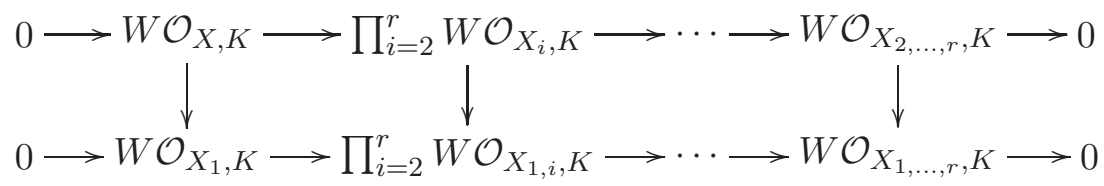

Thus, it suffices to prove that this morphism is a quasi-isomorphism. The induction hypothesis implies that the canonical morphism from the complex $0 \rightarrow W \mathcal{O}_{X, K} \rightarrow W \mathcal{O}_{X^{\prime}, K} \rightarrow 0$ (respectively $0 \rightarrow W \mathcal{O}_{X_{1}, K} \rightarrow W \mathcal{O}_{X_{1} \cap X^{\prime}, K} \rightarrow 0$ ) to the upper line (respectively lower line) of (2.3) is a quasiisomorphism. Therefore, it suffices to prove that the morphism of complexes

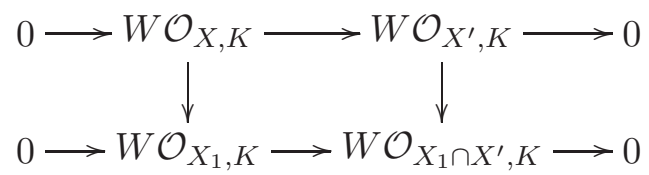

is a quasi-isomorphism, and this follows from Proposition 2.2.

Our next theorem is the main result which allows to use the groups $H^{*}\left(X, W \mathcal{I}_{K}\right)$ to define a Witt vector cohomology with compact supports for separated $k$-schemes of finite type. Its starting point is Deligne's 'independence on the compactification' result in the construction of the $f$ ! functor for coherent sheaves [Har66, Appendix, Proposition 5].

Theorem 2.4. Let $f: X^{\prime} \rightarrow X$ be a proper morphism between two $k$-schemes of finite type, and let $\mathcal{I} \subset \mathcal{O}_{X}$ be a coherent ideal, $\mathcal{I}^{\prime}=\mathcal{I O}_{X^{\prime}}, Y=V(\mathcal{I}), U=X \backslash Y, U^{\prime}=f^{-1}(U)$.

(i) If $f$ induces a finite morphism $U^{\prime} \rightarrow U$, then, for all $q \geqslant 1$,

$$
R^{q} f_{*}\left(W \mathcal{I}_{K}^{\prime}\right)=0 .
$$

(ii) If $f$ induces an isomorphism $U^{\prime} \stackrel{\sim}{\longrightarrow} U$, the canonical morphism

$$
W \mathcal{I}_{K} \rightarrow f_{*}\left(W \mathcal{I}_{K}^{\prime}\right)
$$

is an isomorphism, and induces an isomorphism

$$
\mathbb{R} \Gamma\left(X, W \mathcal{I}_{K}\right) \stackrel{\sim}{\longrightarrow} \mathbb{R} \Gamma\left(X^{\prime}, W \mathcal{I}_{K}^{\prime}\right) .
$$

The proof will use the following general lemma.

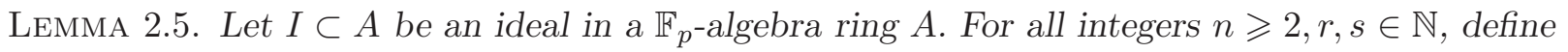

$$
W_{n}^{r, s}(I)=\left\{\left(a_{0}, a_{1}, \ldots\right) \in W_{n}(A) \mid a_{0} \in I^{r}, a_{i} \in I^{s} \text { for all } i \geqslant 1\right\} .
$$




\section{WiTT VECTOR COHOMOLOGY OF SINGULAR VARIETIES}

(i) If $s \leqslant p r$, the subset $W_{n}^{r, s}(I)$ is an ideal of $W_{n}(A)$, which sits in the short exact sequence of abelian groups

$$
0 \rightarrow W_{n-1}\left(I^{s}\right) \stackrel{V}{\longrightarrow} W_{n}^{r, s}(I) \stackrel{R^{n-1}}{\longrightarrow} I^{r} \rightarrow 0 .
$$

(ii) If $r \leqslant s \leqslant p r$, the sequence (2.7) sits in a commutative diagram of short exact sequences

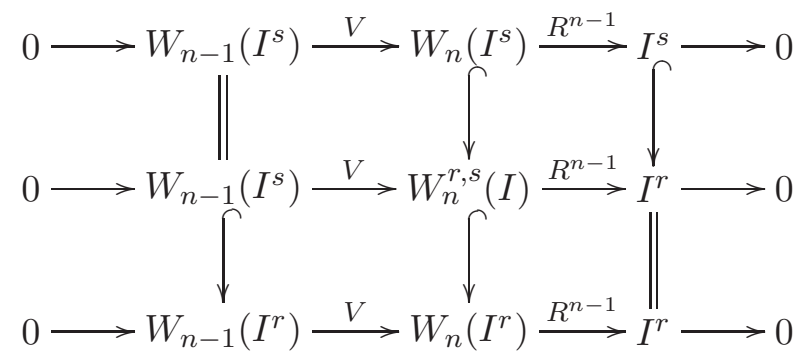

where the vertical arrows are the natural inclusions.

Proof. Assume first that $s \leqslant p r$. The subset $W_{n}^{r, s}(I)$ can be described as the set of Witt vectors of the form $\underline{a}+V(b)$, where $a \in I^{r}, b \in W_{n-1}\left(I^{s}\right)$, and $\underline{a}$ denotes the Teichmüller representative of $a$. To prove that it is an additive subgroup, it suffices to verify that if $a, a^{\prime} \in I^{r}$, then $\underline{a}+\underline{a}^{\prime}=$ $\underline{a+a^{\prime}}+V(c)$, with $c \in W_{n-1}\left(I^{s}\right)$. If $S_{i}\left(X_{0}, \ldots, X_{i}, Y_{0}, \ldots, Y_{i}\right)$ are the universal polynomials defining the addition in $W_{n}(A)$, it is easy to check that $S_{i}\left(X_{0}, 0, \ldots, Y_{0}, 0, \ldots\right)$ is a homogeneous polynomial of degree $p^{i}$ in $\mathbb{Z}\left[X_{0}, Y_{0}\right]$ (see [Ser62, II $\left.\S 6\right]$ ). Since $p r \geqslant s$, the claim follows. Using again the condition $p r \geqslant s$, the fact that $W_{n}^{r, s}(I)$ satisfies the multiplicativity property of an ideal follows from the relations

$$
\underline{a}\left(x_{0}, x_{1}, x_{2}, \ldots\right)=\left(a x_{0}, a^{p} x_{1}, a^{p^{2}} x_{2}, \ldots\right), \quad V(b) x=V(b F(x)) .
$$

If we assume in addition that $r \leqslant s$, then the vertical inclusions of the diagram are defined, and its commutativity is obvious.

We will use the following elementary remark repeatedly.

Lemma 2.6. Let $\mathcal{C}$ be an abelian category, and let

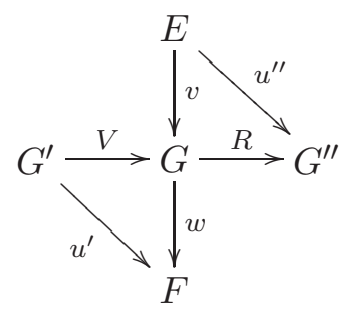

be a commutative diagram of morphisms of $\mathcal{C}$ such that the horizontal sequence is exact. If $u^{\prime}=0$ and $u^{\prime \prime}=0$, then $w \circ v=0$.

Proof. We leave the proof as an exercise for the reader.

LEMma 2.7. (i) Under the assumptions of Theorem 2.4(i), there exists an integer $a \geqslant 0$ such that, for all $q \geqslant 1$, all $n \geqslant 1$, and all $r \geqslant 0$, the canonical morphism

$$
R^{q} f_{*}\left(W_{n}\left(\mathcal{I}^{\prime r+a}\right)\right) \rightarrow R^{q} f_{*}\left(W_{n}\left(\mathcal{I}^{\prime r}\right)\right)
$$

is the zero morphism.

(ii) Assume that $f$ induces an isomorphism $U^{\prime} \stackrel{\sim}{\longrightarrow} U$, and define

$$
\begin{aligned}
\mathcal{K}_{n}^{r} & =\operatorname{Ker}\left(W_{n}\left(\mathcal{I}^{r}\right) \rightarrow f_{*}\left(W_{n}\left(\mathcal{I}^{\prime r}\right)\right)\right), \\
\mathcal{C}_{n}^{r} & =\operatorname{Coker}\left(W_{n}\left(\mathcal{I}^{r}\right) \rightarrow f_{*}\left(W_{n}\left(\mathcal{I}^{\prime r}\right)\right)\right) .
\end{aligned}
$$




\section{P. Berthelot, S. Bloch and H. Esnault}

Then there exists an integer $a \geqslant 0$ such that, for all $n \geqslant 1$ and all $r \geqslant 0$, the canonical morphisms

$$
\mathcal{K}_{n}^{r+a} \rightarrow \mathcal{K}_{n}^{r}, \quad \mathcal{C}_{n}^{r+a} \rightarrow \mathcal{C}_{n}^{r},
$$

are the zero morphisms.

Proof. We first prove assertion (i). We may fix $q \geqslant 1$, since $R^{q} f_{*}=0$ for $q$ big enough. When $n=1$, we can apply Deligne's result [Har66, Appendix, Proposition 5] to $\mathcal{O}_{X^{\prime}}$, and we obtain an integer $b \geqslant 0$ such that, for all $r \geqslant 0$, the canonical morphism

$$
u_{1}: R^{q} f_{*}\left(\mathcal{I}^{\prime r+b}\right) \rightarrow R^{q} f_{*}\left(\mathcal{I}^{\prime r}\right)
$$

is 0 (note that, for this result, Deligne's argument only uses that $f$ is finite above $U$ ). Let us prove by induction on $n$ that, for all $n \geqslant 1$ and all $r \geqslant b$, the canonical morphism

$$
u_{n}: R^{q} f_{*}\left(W_{n}\left(\mathcal{I}^{\prime r+b}\right)\right) \rightarrow R^{q} f_{*}\left(W_{n}\left(\mathcal{I}^{\prime r}\right)\right)
$$

is also 0 .

The condition $r \geqslant b$ implies that the couple $(r, r+b)$ is such that $r \leqslant r+b \leqslant p r$. Therefore, we may use Lemma 2.5 to define ideals $W_{n}^{r, r+b}\left(\mathcal{I}^{\prime}\right) \subset W_{n}\left(\mathcal{O}_{X^{\prime}}\right)$ and, for $n \geqslant 2$, we obtain a commutative diagram (2.8) relative to $\mathcal{I}^{\prime}$ and $(r, r+b)$. Since the middle row of $(2.8)$ is a short exact sequence, the diagram

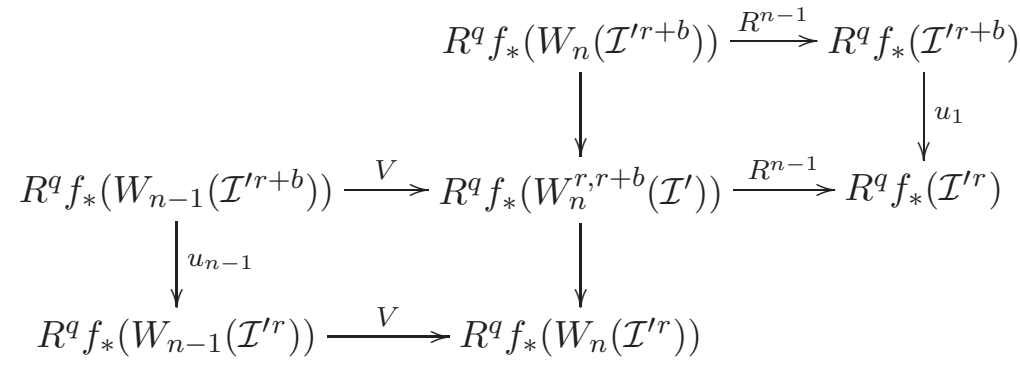

obtained by applying the functor $R^{q} f_{*}$ to $(2.8)$ has an exact middle row. As $u_{1}=0$, and the composition of the middle vertical arrows is $u_{n}$, Lemma 2.6 shows by induction that $u_{n}=0$ for all $n \geqslant 1$. If we set $a=2 b$, then assertion (i) holds.

Assertion (ii) can be proved by the same method. Let $\mathcal{R}=\bigoplus_{r \geqslant 0} \mathcal{I}^{r}$. It follows from [Gro61, Proposition 3.3.1] that the quasi-coherent graded $\mathcal{R}$-modules $\mathcal{K}_{1}=\bigoplus_{r \geqslant 0} \mathcal{K}_{1}^{r}$ and $\mathcal{C}_{1}=\bigoplus_{r \geqslant 0} \mathcal{C}_{1}^{r}$ are finitely generated. As the restriction of $f$ to $U^{\prime}$ is an isomorphism, they are supported in $Y$. Therefore, there exists an integer $m$ such that $\mathcal{I}^{m} \mathcal{K}_{1}^{r}=\mathcal{I}^{m} \mathcal{C}_{1}^{r}=0$ for all $r \geqslant 0$. Moreover, there exists an integer $d$ such that, for all $r \geqslant d$ the canonical morphisms

$$
\mathcal{I} \otimes \mathcal{O}_{X} \mathcal{K}_{1}^{r} \rightarrow \mathcal{K}_{1}^{r+1}, \quad \mathcal{I} \otimes \mathcal{O}_{X} \mathcal{C}_{1}^{r} \rightarrow \mathcal{C}_{1}^{r+1},
$$

are surjective. It follows that the morphisms

$$
\mathcal{K}_{1}^{r+m} \rightarrow \mathcal{K}_{1}^{r}, \quad \mathcal{C}_{1}^{r+m} \rightarrow \mathcal{C}_{1}^{r}
$$

are 0 for $r \geqslant d$. Replacing $m$ by $b=d+m$, the corresponding morphisms are 0 for all $r \geqslant 0$, which proves assertion (ii) when $n=1$.

As $\mathcal{K}_{1}^{r} \subset \mathcal{O}_{X}$ for all $r$, this implies that $\mathcal{K}_{1}^{r}=0$ for $r \geqslant b$. Thanks to the exact sequences

$$
0 \rightarrow \mathcal{K}_{n-1}^{r} \stackrel{V}{\longrightarrow} \mathcal{K}_{n}^{r} \stackrel{R^{n-1}}{\longrightarrow} \mathcal{K}_{1}^{r},
$$

it follows that $\mathcal{K}_{n}^{r}=0$ for all $n \geqslant 1$ and all $r \geqslant b$. Thus, assertion (ii) holds for the modules $\mathcal{K}_{n}^{r}$, with $a=b$.

To prove it for the modules $\mathcal{C}_{n}^{r}$, we introduce for $r \geqslant b$ the modules

$$
\mathcal{C}_{n}^{r, r+b}=\operatorname{Coker}\left(W_{n}^{r, r+b}(\mathcal{I}) \rightarrow f_{*}\left(W_{n}^{r, r+b}\left(\mathcal{I}^{\prime}\right)\right)\right) .
$$




\section{WiTT VECTOR COHOMOLOGY OF SINGULAR VARIETIES}

The snake lemma applied to the diagrams

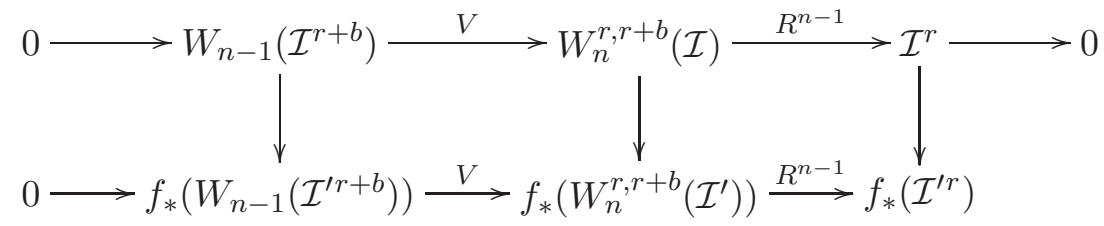

gives exact sequences

$$
\mathcal{C}_{n-1}^{r+b} \stackrel{V}{\longrightarrow} \mathcal{C}_{n}^{r, r+b} \stackrel{R^{n-1}}{\longrightarrow} \mathcal{C}_{1}^{r}
$$

The functoriality of the diagrams (2.8) imply that these exact sequences sit in commutative diagrams

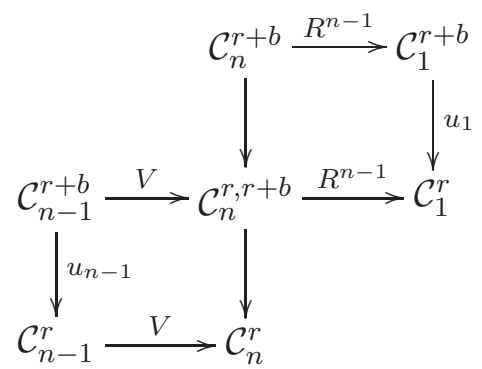

where the morphisms $u_{i}$ are the canonical morphisms, and the composition of the middle vertical arrows is $u_{n}$. As $u_{1}=0$, Lemma 2.6 implies by induction that $u_{n}=0$ for all $n$. This proves assertion (ii) for $\mathcal{C}_{n}^{r}$, with $a=2 b$.

2.8 Proof of Theorem 2.4. Under the assumptions of Theorem 2.4, let $q \geqslant 1$ be an integer, and let $a$ be an integer satisfying the conclusion of Lemma 2.7(i) for the family of sheaves $R^{q} f_{*}\left(W_{n}\left(\mathcal{I}^{\prime r}\right)\right)$, for all $n \geqslant 1$ and $r \geqslant 0$. Let $c$ be such that $p^{c}>a$. Since the Frobenius map $F^{c}: R^{q} f_{*}\left(W_{n}\left(\mathcal{I}^{\prime r}\right)\right) \rightarrow$ $R^{q} f_{*}\left(W_{n}\left(\mathcal{I}^{\prime r}\right)\right)$ factors through $R^{q} f_{*}\left(W_{n}\left(\mathcal{I}^{p^{c} r}\right)\right)$, it follows from Lemma 2.7 that $F^{c}$ acts by zero on $R^{q} f_{*}\left(W_{n}\left(\mathcal{I}^{\prime r}\right)\right)$, for all $n \geqslant 1$ and all $r \geqslant 1$. Therefore, for all $r \geqslant 1, F^{c}$ acts by zero on $\mathbb{R} \lim _{n}\left(R^{q} f_{*}\left(W_{n}\left(\mathcal{I}^{\prime r}\right)\right)\right)$.

In particular, $F^{c}$ acts by zero on the sheaves $\lim _{n} R^{q} f_{*}\left(W_{n} \mathcal{I}^{\prime}\right)$ and $R^{1} \varliminf_{n} R^{q} f_{*}\left(W_{n} \mathcal{I}^{\prime}\right)$. As the inverse system $\left(W_{n} \mathcal{I}^{\prime}\right)_{n \geqslant 1}$ has surjective transition maps, and terms with vanishing cohomology on affine open subsets, it is $\lim _{n}$-acyclic, and we obtain

$$
\mathbb{R} f_{*}\left(W \mathcal{I}^{\prime}\right)=\mathbb{R} f_{*}\left(\underset{\mathbb{R}}{\lim _{n}} W_{n} \mathcal{I}^{\prime}\right) \cong \mathbb{R}{\underset{n}{\lim }}_{\mathbb{R}} f_{*}\left(W_{n} \mathcal{I}^{\prime}\right) .
$$

This isomorphism provides a biregular spectral sequence

$$
E_{2}^{i, j}=R^{i} \varliminf_{n} R^{j} f_{*}\left(W_{n} \mathcal{I}^{\prime}\right) \Rightarrow R^{i+j} f_{*}\left(W \mathcal{I}^{\prime}\right)
$$

in which the filtration of the terms $R^{i+j} f_{*}\left(W \mathcal{I}^{\prime}\right)$ is of length at most 2 since the functors $R^{i} \varliminf_{n}$ are zero for $i \geqslant 2$. As $F^{c}$ acts by zero on the terms $E_{2}^{i, j}$ for $j \geqslant 1, F^{2 c}$ acts by zero on the terms $R^{i+j} f_{*}\left(W \mathcal{I}^{\prime}\right)$ for $i+j \geqslant 2$.

On the other hand, the term $E_{2}^{1,0}=R^{1} \varliminf_{n} f_{*}\left(W_{n} \mathcal{I}^{\prime}\right)$ is 0 , because the morphisms $f_{*}\left(W_{n+1} \mathcal{I}^{\prime}\right) \rightarrow$ $f_{*}\left(W_{n} \mathcal{I}^{\prime}\right)$ are surjective (since $f_{*}\left(W_{n} \mathcal{I}^{\prime}\right) \cong W_{n}^{n}\left(f_{*}\left(\mathcal{I}^{\prime}\right)\right)$ ), and the cohomology of the terms $f_{*}\left(W_{n} \mathcal{I}^{\prime}\right)$ vanishes on any open affine subset. Therefore, $F^{c}$ acts by zero on $R^{1} f_{*}\left(W \mathcal{I}^{\prime}\right)$.

Thus the action of $F$ on $R^{q} f_{*}\left(W \mathcal{I}^{\prime}\right)$ is nilpotent for all $q \geqslant 1$. Since this action becomes an isomorphism after tensorization with $K$, we obtain that $R^{q} f_{*}\left(W \mathcal{I}_{K}^{\prime}\right)=R^{q} f_{*}\left(W \mathcal{I}^{\prime}\right)_{K}=0$ for all $q \geqslant 1$, which proves assertion (i) of Theorem 2.4. 


\section{P. Berthelot, S. Bloch and H. Esnault}

Let us assume now that $f: U^{\prime} \rightarrow U$ is an isomorphism. It follows from Lemma 2.7(ii) that there exists an integer $a$ such that, for all $n \geqslant 1$ and all $r \geqslant 0$, the morphisms $\mathcal{K}_{n}^{r+a} \rightarrow \mathcal{K}_{n}^{r}$ and $\mathcal{C}_{n}^{r+a} \rightarrow \mathcal{C}_{n}^{r}$ are zero. Taking $c$ such that $p^{c}>a$, it follows that $F^{c}$ acts by zero on $\mathcal{K}_{n}^{r}$ and $\mathcal{C}_{n}^{r}$ for all $n$ and all $r$. Therefore, $F^{c}$ acts by zero on $\varliminf_{n} \mathcal{K}_{n}^{1}$ and $\lim _{n} \mathcal{C}_{n}^{1}$. It is easy to check that these two inverse systems and the inverse system $\operatorname{Im}\left({\overleftarrow{W_{n}}}^{n} \mathcal{I} \rightarrow f_{*}\left({\overleftarrow{W_{n} \mathcal{I}^{\prime}}}^{n}\right)\right.$ all have surjective transition maps. As their terms have vanishing cohomology on affine open subsets, they are $\varliminf_{\longleftarrow}$-acyclic, and we obtain

$$
\begin{gathered}
\operatorname{Ker}\left(W \mathcal{I} \rightarrow f_{*}\left(W \mathcal{I}^{\prime}\right)\right)={\underset{\bigsqcup}{n}}_{\lim } \mathcal{K}_{n}^{1}, \\
\operatorname{Coker}\left(W \mathcal{I} \rightarrow f_{*}\left(W \mathcal{I}^{\prime}\right)\right)={\underset{\lim }{n} \mathcal{C}_{n}^{1} .}^{\operatorname{lin}}
\end{gathered}
$$

After tensorization with $K, F$ becomes an isomorphism on $\varliminf_{n} \mathcal{K}_{n}^{1}$ and $\varliminf_{n} \mathcal{L}_{n}^{1}$, and assertion (ii) of Theorem 2.4 follows.

2.9 We now observe that the previous results imply that the cohomology spaces $H^{*}\left(X, W \mathcal{I}_{K}\right)$ only depend on the $k$-scheme $U$, and have the same functoriality properties with respect to $U$ as cohomology with compact supports.

Indeed, suppose that $U$ is fixed, and let $U \hookrightarrow X_{1}, U \hookrightarrow X_{2}$ be two open immersions into proper $k$-schemes. Let $X \subset X_{1} \times_{k} X_{2}$ be the scheme theoretic closure of $U$ embedded diagonally into $X_{1} \times_{k} X_{2}$. The two projections induce proper maps $p_{1}: X \rightarrow X_{1}, p_{2}: X \rightarrow X_{2}$. If $\mathcal{I} \subset \mathcal{O}_{X}$, $\mathcal{I}_{1} \subset \mathcal{O}_{X_{1}}, \mathcal{I}_{2} \subset \mathcal{O}_{X_{2}}$ are the ideals defining $Y=(X \backslash U)_{\text {red }}, Y_{1}=\left(X_{1} \backslash U\right)_{\text {red }}$, and $Y_{2}=\left(X_{2} \backslash U\right)_{\text {red }}$, we deduce from Proposition 2.1 and Theorem 2.4 that the homomorphisms

$$
\mathbb{R} \Gamma\left(X_{2}, W \mathcal{I}_{2, K}\right) \stackrel{p_{2}^{*}}{\longrightarrow} \mathbb{R} \Gamma\left(X, W \mathcal{I}_{K}\right) \stackrel{p_{1}^{*}}{\longleftarrow} \mathbb{R} \Gamma\left(X_{1}, W \mathcal{I}_{1, K}\right)
$$

are isomorphisms. If we define

$$
\varepsilon_{12}=p_{1}^{*-1} \circ p_{2}^{*}: \mathbb{R} \Gamma\left(X_{2}, W \mathcal{I}_{2, K}\right) \stackrel{\sim}{\longrightarrow} \mathbb{R} \Gamma\left(X_{1}, W \mathcal{I}_{1, K}\right),
$$

it is easy to check that the isomorphisms $\varepsilon_{i j}$ satisfy the transitivity condition for a third open immersion $U \hookrightarrow X_{3}$ into a proper $k$-scheme. Therefore, they provide canonical identifications between the cohomology complexes $\mathbb{R} \Gamma\left(X, W \mathcal{I}_{K}\right)$ defined by various open immersions of $U$ into proper $k$-schemes $X$.

Assume that $U$ is a separated $k$-scheme of finite type. Since, by Nagata's theorem, there exists a proper $k$-scheme $X$ and an open immersion $U \hookrightarrow X$, this independence property allows us to define the Witt vector cohomology with compact supports of $U$ by setting

$$
\begin{aligned}
\mathbb{R} \Gamma_{\mathrm{c}}\left(U, W \mathcal{O}_{U, K}\right) & :=\mathbb{R} \Gamma\left(X, W \mathcal{I}_{K}\right) \cong \mathbb{R} \Gamma(X, W \mathcal{I})_{K}, \\
H_{\mathrm{c}}^{*}\left(U, W \mathcal{O}_{U, K}\right) & :=H^{*}\left(X, W \mathcal{I}_{K}\right) \cong H^{*}(X, W \mathcal{I})_{K},
\end{aligned}
$$

where $\mathcal{I} \subset \mathcal{O}_{X}$ is any coherent ideal defining the closed subset $X \backslash U$. As the restriction of $W \mathcal{I}$ to $U$ is $W \mathcal{O}_{U}$, there is a canonical morphism $\mathbb{R} \Gamma_{\mathrm{c}}\left(U, W \mathcal{O}_{U, K}\right) \rightarrow \mathbb{R} \Gamma\left(U, W \mathcal{O}_{U, K}\right)$, which is an isomorphism when $U$ is proper.

These cohomology groups have the following functoriality properties.

(i) They are contravariant with respect to proper maps.

Let $f: U^{\prime} \rightarrow U$ be a proper $k$-morphism of separated $k$-schemes, and let $U^{\prime} \hookrightarrow X^{\prime}, U \hookrightarrow X$ be open immersions into proper $k$-schemes. Replacing, if necessary, $X^{\prime}$ by the scheme theoretic closure of the graph of $f$ in $X^{\prime} \times_{k} X$, we may assume that there exists a $k$-morphism $g: X^{\prime} \rightarrow X$ extending $f$, and that $U^{\prime}$ is dense in $X^{\prime}$. As $f$ is proper, it follows that $U^{\prime}=g^{-1}(U)$. If $\mathcal{I} \subset \mathcal{O}_{X}$ is any coherent ideal such that $V(\mathcal{I})=X \backslash U$, then $\mathcal{I}^{\prime}=\mathcal{I O}_{X^{\prime}}$ is such that $V\left(\mathcal{I}^{\prime}\right)=X^{\prime} \backslash U^{\prime}$, and we 


\section{WiTT VECTOR COHOMOLOGY OF SINGULAR VARIETIES}

can define the homomorphism

$$
f^{*}: \mathbb{R} \Gamma_{\mathrm{c}}\left(U, W \mathcal{O}_{U, K}\right) \rightarrow \mathbb{R} \Gamma_{\mathrm{c}}\left(U^{\prime}, W \mathcal{O}_{U^{\prime}, K}\right)
$$

as being

$$
g^{*}: \mathbb{R} \Gamma\left(X, W \mathcal{I}_{K}\right) \rightarrow \mathbb{R} \Gamma\left(X^{\prime}, W \mathcal{I}_{K}^{\prime}\right) .
$$

We leave it as an exercise for the reader to check that, up to canonical isomorphism, $f^{*}$ does not depend on the choices.

(ii) They are covariant with respect to open immersions.

Let $j: V \hookrightarrow U$ be an open immersion, let $U \hookrightarrow X$ be an open immersion into a proper $k$-scheme, and let $\mathcal{I}, \mathcal{J} \subset \mathcal{O}_{X}$ be the ideals of $Y=(X \backslash U)_{\text {red }}$ and $Z=(X \backslash V)_{\text {red. }}$ Then the homomorphism

$$
j_{*}: \mathbb{R} \Gamma_{\mathrm{c}}\left(V, W \mathcal{O}_{V, K}\right) \rightarrow \mathbb{R} \Gamma_{\mathrm{c}}\left(U, W \mathcal{O}_{U, K}\right)
$$

is defined as being

$$
\mathbb{R} \Gamma\left(X, W \mathcal{J}_{K}\right) \rightarrow \mathbb{R} \Gamma\left(X, W \mathcal{I}_{K}\right)
$$

If $T=U \backslash V=Z \cap U$, then $T$ is open in the proper $k$-scheme $Z$, and the ideal $\mathcal{I} / \mathcal{J} \subset \mathcal{O}_{Z}$ defines the complement of $T$ in $Z$. Thus, the usual distinguished triangle

$$
\mathbb{R} \Gamma_{\mathrm{c}}\left(V, W \mathcal{O}_{V, K}\right) \rightarrow \mathbb{R} \Gamma_{\mathrm{c}}\left(U, W \mathcal{O}_{U, K}\right) \rightarrow \mathbb{R} \Gamma_{\mathrm{c}}\left(T, W \mathcal{O}_{T, K}\right) \stackrel{+1}{\longrightarrow}
$$

is obtained by tensoring the short exact sequence

$$
0 \rightarrow W \mathcal{J} \rightarrow W \mathcal{I} \rightarrow W(\mathcal{I} / \mathcal{J}) \rightarrow 0
$$

with $K$, and taking its cohomology on $X$.

Proposition 2.10. Let $U$ be a separated $k$-scheme of finite type. Then the cohomology spaces $H_{\mathrm{c}}^{*}\left(U, W \mathcal{O}_{U, K}\right)$ are finite-dimensional $K$-vector spaces, on which the Frobenius endomorphism has slopes in $[0,1[$.

Proof. It suffices to prove the statement when $U$ is a proper $k$-scheme $X$. Then the statement reduces to the finiteness of the usual cohomology spaces $H^{*}\left(X, W \mathcal{O}_{X}\right)_{K}$. This is a well-known result (cf. [Blo77, III, Theorem 2.2], which is valid for $W \mathcal{O}_{X}$ without the smoothness assumption). For the sake of completeness, we give a proof here.

Write $M:=H^{i}\left(X, W \mathcal{O}_{X}\right)$. As $X$ is proper, the groups $H^{i}\left(X, W_{n} \mathcal{O}_{X}\right)$ satisfy the Mittag-Leffler condition, so that the homomorphism

$$
H^{i}\left(X, W \mathcal{O}_{X}\right) \rightarrow \underset{n}{\lim _{n}} H^{i}\left(X, W_{n} \mathcal{O}_{X}\right)
$$

is an isomorphism. Therefore, the Verschiebung endomorphism endows $M$ with a structure of module over the non-necessarily commutative ring $R:=W_{\sigma}[[V]]$, where the index $\sigma$ refers to the commutation rules $a V=V \sigma(a)$ for $a \in W$. Then $M$ is separated and complete for the $V$-adic topology. On the other hand, $M / V M \hookrightarrow H^{i}\left(X, \mathcal{O}_{X}\right)$ is a finite-dimensional $k$-vector space. Since $M / V M$ is finitely generated over $W$, it follows that $M$ is finitely generated over $R$. Moreover, the fact that $M / V M$ has finite length implies that $M$ is a torsion $R$-module. Thus, there exists a finite number of non-zero elements $a_{i}(V)=a_{i, 0}+a_{i, 1} V+a_{i, 2} V^{2}+\cdots \in R, i=1, \ldots, r$, and a surjection

$$
\bigoplus_{i=1}^{r} R / R a_{i}(V) \rightarrow M .
$$

Fix some $i, 1 \leqslant i \leqslant r$. We are interested in the module structure after inverting $p$, so we may assume that $a_{i, \ell} \in W(k)^{\times}$for some $\ell$. Let $\ell$ be minimal, so $p \mid a_{i, j}, 0 \leqslant j<\ell$. Even if the $\operatorname{ring} R$ is not 


\section{P. Berthelot, S. Bloch and H. Esnault}

commutative, we can get a factorization

$$
\sum_{j} a_{i, j} V^{j}=\left(c_{i, 0}+c_{i, 1} V+\cdots\right)\left(b_{i, 0}+\cdots+b_{i, \ell-1} V^{\ell-1}+V^{\ell}\right) ; \quad p \mid b_{i, j} .
$$

To see this, we factor

$$
\sum_{j} a_{i, j} V^{j} \equiv\left(c_{i, 0}^{(s)}+c_{i, 1}^{(s)} V+\cdots\right)\left(b_{i, 0}^{(s)}+\cdots+b_{i, \ell-1}^{(s)} V^{\ell-1}+V^{\ell}\right) \quad \bmod p^{s+1},
$$

where $b_{i, j}^{(s+1)}, c_{i, j}^{(s+1)} \equiv b_{i, j}^{(s)}, c_{i, j}^{(s)} \bmod p^{s+1}$, starting with $b_{i, j}^{(0)}=0$ and $c_{i, j}^{(0)}=a_{i, j+\ell}$. Note that $c_{i, 0}^{(s)} \in$ $W(k)^{\times}$. Details are standard and are left for the reader. Writing $b_{i}(V)=b_{i, 0}+b_{i, 1} V+\cdots+V^{\ell}$ with $b_{i, j}=\lim b_{i, j}^{(s)}$, it follows that $R / R a_{i}(V) \cong R / R b_{i}(V) \cong W(k)^{\oplus \ell}$. The finiteness now follows from $(2.15)$.

As $H^{*}\left(X, W \mathcal{O}_{X}\right)$ is a finitely generated $W$-module modulo torsion, the slopes of the Frobenius endomorphism are positive. On the other hand, the existence of a Verschiebung endomorphism $V$ such that $F V=V F=p$ implies that all slopes are at most 1 . As $V$ is topologically nilpotent, there cannot be any non-zero element of slope 1 in $H^{*}\left(X, W \mathcal{O}_{X, K}\right)$, and all its slopes belong to $[0,1[$.

\section{A descent theorem}

We need simplicial resolutions based on de Jong's fundamental result on alterations. In this section, we briefly recall some related definitions, and how to construct such resolutions. We then prove for Witt vector cohomology with compact supports a particular case of étale cohomological descent which will be one of the main ingredients in our proof of Theorem 1.1.

If $X$ is a scheme, and $n \geqslant-1$ an integer, we denote as usual by $\operatorname{sk}_{n}^{X}$ the truncation functor from the category of simplicial $X$-schemes to the category of $n$-truncated simplicial $X$-schemes, and by $\operatorname{cosk}_{n}^{X}$ its right adjoint [Del74, $\left.\S 5.1\right]$.

Definition 3.1. Let $X$ be a reduced $k$-scheme of finite type, and let $X$. be a simplicial $k$-scheme (respectively $N$-truncated simplicial scheme, for some $N \in \mathbb{N}$ ).

(a) A $k$-augmentation $f_{\bullet}: X_{\bullet} \rightarrow X$ is called a proper hypercovering (respectively $N$-truncated proper hypercovering) of $X$ if the canonical morphism $X_{n} \rightarrow \operatorname{cosk}_{n-1}^{X}\left(\operatorname{sk}_{n-1}^{X}\left(X_{\bullet}\right)\right)_{n}$ is proper and surjective for all $n \geqslant 0$ (respectively $0 \leqslant n \leqslant N$ ).

(b) A $k$-augmentation $f_{\bullet}: X_{\bullet} \rightarrow X$ is called an étale hypercovering (respectively $N$-truncated étale hypercovering) of $X$ if the canonical morphism $X_{n} \rightarrow \operatorname{cosk}_{n-1}^{X}\left(\operatorname{sk}_{n-1}^{X}\left(X_{\bullet}\right)\right)_{n}$ is étale and surjective for all $n \geqslant 0$ (respectively $0 \leqslant n \leqslant N$ ).

(c) A $k$-augmentation $f_{\bullet}: X_{\bullet} \rightarrow X$ is called a simplicial resolution (respectively $N$-truncated simplicial resolution) of $X$ if the following conditions hold:

(i) $f_{\bullet}$ is a proper hypercovering (respectively $N$-truncated proper hypercovering) of $X$;

(ii) for all $n \geqslant 0$ (respectively $0 \leqslant n \leqslant N$ ), there exists a dense open subset $U_{n} \subset X$ such that the restriction of $\operatorname{sk}_{n}^{X}\left(X_{\bullet}\right)$ above $U_{n}$ is an $n$-truncated étale hypercovering of $U_{n}$;

(iii) for all $n \geqslant 0$ (respectively $0 \leqslant n \leqslant N$ ), $X_{n}$ is a smooth quasi-projective $k$-scheme.

Since $k$ is perfect, de Jong's theorem [Dej96, Theorem 4.1 and Remark 4.2] implies that, for any separated integral (i.e. reduced and irreducible) $k$-scheme of finite type $X$, there exists a quasiprojective, smooth, and integral $k$-scheme $X^{\prime}$, and a surjective, projective, and generically étale morphism $X^{\prime} \rightarrow X$. If $X$ is separated and reduced, but not necessarily irreducible, one can apply de Jong's theorem to each irreducible component of $X$, and in this way one obtains a quasi-projective smooth $k$-scheme $X^{\prime}$, and a surjective projective morphism $X^{\prime} \rightarrow X$ which is étale over a dense 


\section{WitT VECTOR COHOMOLOGY OF SINGULAR VARIETIES}

open subset of $X$. Replacing the resolution of singularities by this result, one can then proceed as Deligne [Del74, $\S 6.2 .5]$ to show that any separated reduced $k$-scheme of finite type has a simplicial resolution.

Remark. Let $\mathcal{C}$ be the category of $X$-schemes which are separated of finite type over $k$, and $\mathcal{D}$ the subcategory defined as follows:

- $\mathrm{Ob}(\mathcal{D})=\mathrm{Ob}(\mathcal{C})$

- if $Y, Z \in \operatorname{Ob}(\mathcal{D}), \operatorname{Hom}_{\mathcal{D}}(Y, Z)$ is the set of $X$-morphisms $f: Y \rightarrow Z$ which are proper, surjective, and such that there exists a dense open subset $U \subset X$ with the property that the restriction $\left.f\right|_{U}:\left.\left.Y\right|_{U} \rightarrow Z\right|_{U}$ of $f$ above $U$ is étale.

Then $\mathcal{D}$ satisfies the condition $(\mathrm{HC})$ of $[\mathrm{Tsu} 04, \S 5.1]$, and the simplicial resolutions of $X$ are the $\mathcal{D}$ hypercoverings of $X$ by smooth quasi-projective $k$-schemes, in the sense of [Tsu04, Definition 5.1.1].

Proposition 3.2. Let $X$ be a reduced $k$-scheme of finite type, let $N \in \mathbb{N}$, and let $f_{\bullet}: X_{\bullet} \rightarrow X$ be an $N$-truncated proper hypercovering of $X$. Let $\mathcal{I} \subset \mathcal{O}_{X}$ be a coherent ideal, $\mathcal{I}_{q}=\mathcal{I O}_{X_{q}}, Y=V(\mathcal{I})$, $U=X \backslash Y, U_{q}=f_{q}^{-1}(U)$. Assume that the restriction $U$. of $X$. above $U$ is an $N$-truncated étale hypercovering of $U$. Then:

(i) the complex

$$
0 \rightarrow W \mathcal{I}_{K} \rightarrow f_{0 *}\left(W \mathcal{I}_{0, K}\right) \rightarrow f_{1 *}\left(W \mathcal{I}_{1, K}\right) \rightarrow \cdots \rightarrow f_{N *}\left(W \mathcal{I}_{N, K}\right),
$$

where $W \mathcal{I}_{K}$ sits in degree -1, is acyclic in degrees not equal to $N$;

(ii) the canonical morphisms

$$
H^{q}\left(X, W \mathcal{I}_{K}\right) \rightarrow H^{q}\left(X_{\bullet}, W \mathcal{I}_{\bullet}, K\right)
$$

are isomorphisms for all $q<N$.

Proof. For each $n \geqslant 1$ and each $r \geqslant 0$, we denote by $\mathcal{L}_{n}^{\bullet}, r$ the complex

$$
0 \rightarrow W_{n}\left(\mathcal{I}^{r}\right) \rightarrow f_{0 *}\left(W_{n}\left(\mathcal{I}_{0}^{r}\right)\right) \rightarrow \cdots \rightarrow f_{N *}\left(W_{n}\left(\mathcal{I}_{N}^{r}\right)\right),
$$

where $W_{n}\left(\mathcal{I}^{r}\right)$ sits in degree -1 , and we set

$$
\begin{gathered}
\mathcal{L}_{n}^{\bullet}=\bigoplus_{r \geqslant 0} \mathcal{L}_{n}^{\bullet, r}, \\
\mathcal{H}_{n}^{q, r}=\mathcal{H}^{q}\left(\mathcal{L}_{n}^{\bullet, r}\right), \quad \mathcal{H}_{n}^{q}=\mathcal{H}^{q}\left(\mathcal{L}_{n}^{\bullet}\right)=\bigoplus_{r \geqslant 0} \mathcal{H}_{n}^{q, r} .
\end{gathered}
$$

If we denote again by $\mathcal{R}$ the graded $\mathcal{O}_{X}$-algebra $\bigoplus_{r \geqslant 0} \mathcal{I}^{r}$, it follows from [Gro61, Proposition 3.3.1] that $\mathcal{L}_{1}^{\bullet}$ is a complex of quasi-coherent graded $\mathcal{R}$-modules of finite type. Therefore, $\mathcal{H}_{1}^{q}$ is a quasi-coherent graded $\mathcal{R}$-module of finite type for all $q$, and there exists an integer $d \geqslant 0$ such that the morphism

$$
\mathcal{I} \otimes \mathcal{O}_{X} \mathcal{H}_{1}^{q, r} \rightarrow \mathcal{H}_{1}^{q, r+1}
$$

is surjective for all $r \geqslant d$. Moreover, $U$. is an $N$-truncated étale hypercovering of $U$, and therefore it satisfies cohomological descent for quasi-coherent modules. It follows that $\left.\mathcal{L}_{1}\right|_{U}$ is acyclic in degrees less than $N$. Then we can find an integer $m \geqslant 0$ such that $\mathcal{I}^{m} \mathcal{H}_{1}^{q, r}=0$ for all $q<N$ and all $r \geqslant 0$. For $r \geqslant d$, this implies that the image of $\mathcal{H}_{1}^{q, r+m}$ in $\mathcal{H}_{1}^{q, r}$ is 0 . Finally, if we set $b=d+m$, we obtain that the canonical morphism $\mathcal{H}_{1}^{q, r+b} \rightarrow \mathcal{H}_{1}^{q, r}$ is 0 for all $q<N$ and all $r \geqslant 0$.

We can now proceed as in the proof of Lemma 2.7 to prove that the morphism $\mathcal{H}_{n}^{q, r+b} \rightarrow \mathcal{H}_{n}^{q, r}$ is 0 for all $q<N$, all $n \geqslant 1$ and all $r \geqslant b$. As this last condition implies that $r \leqslant r+b \leqslant p r$, we can introduce for $n \geqslant 2$ the subcomplex $\mathcal{L}_{n}^{\bullet, r, r+b} \subset \mathcal{L}_{n}^{\bullet, r}$ defined by

$$
0 \rightarrow W_{n}^{r, r+b}(\mathcal{I}) \rightarrow f_{0 *}\left(W_{n}^{r, r+b}\left(\mathcal{I}_{0}\right)\right) \rightarrow \cdots \rightarrow f_{N *}\left(W_{n}^{r, r+b}\left(\mathcal{I}_{N}\right)\right),
$$




\section{P. Berthelot, S. Bloch and H. Esnault}

and $\mathcal{H}_{n}^{q, r, r+b}=\mathcal{H}^{q}\left(\mathcal{L}_{n}^{\bullet, r, r+b}\right)$. From Lemma 2.5, we deduce a commutative diagram of morphisms of complexes

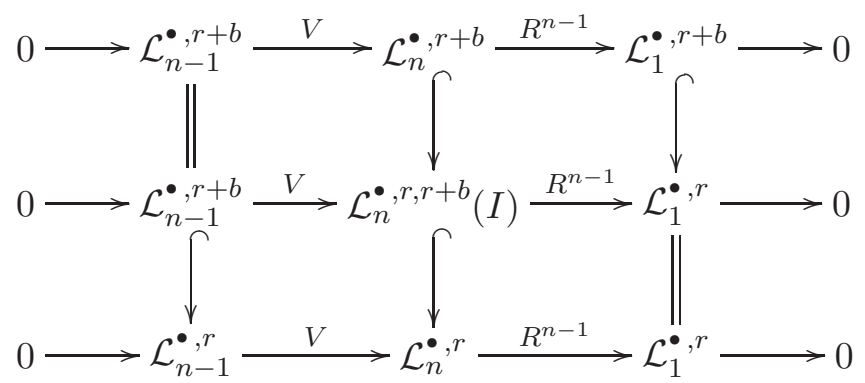

where the rows are short exact sequences, because any section of a sheaf $f_{q *}\left(\mathcal{I}_{q}^{r}\right)$ can be lifted to a section of $f_{q *}\left(W_{n}\left(\mathcal{I}_{q}^{r}\right)\right)$ or $f_{q *}\left(W_{n}^{r, r+b}\left(\mathcal{I}_{q}\right)\right)$ by taking its Teichmüller representative. From the corresponding diagram of cohomology exact sequences, we can extract for each $q$ the commutative diagram

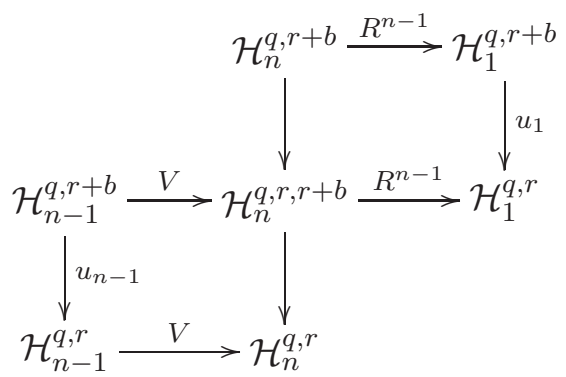

where the morphisms $u_{1}, u_{n-1}$, and the composition of the two vertical morphisms are the canonical morphisms, and the middle row is exact. Since $u_{1}=0$ when $q<N$, Lemma 2.6 shows by induction that $u_{n}=0$ for all $q<N$, all $n \geqslant 1$ and all $r \geqslant b$.

Setting $a=2 b$, we obtain that the morphisms $\mathcal{H}_{n}^{q, r+a} \rightarrow \mathcal{H}_{n}^{q, r}$ are 0 for all $q<N$, all $n \geqslant 1$ and all $r \geqslant 0$. This implies that, if $c$ is chosen so that $p^{c}>a$, then $F^{c}$ acts by 0 on the sheaves $\mathcal{H}_{n}^{q, r}$ for $r \geqslant 1$. Therefore, $F^{c}$ acts by 0 on $R^{i} \lim _{n} \mathcal{H}_{n}^{q, r}$ for all $i \geqslant 0$, all $q<N$ and all $r \geqslant 1$.

Let $\mathcal{L}^{\bullet}$ be the complex

$$
0 \rightarrow W \mathcal{I} \rightarrow f_{0 *}\left(W \mathcal{I}_{0}\right) \rightarrow \cdots \rightarrow f_{N *}\left(W \mathcal{I}_{N}\right)
$$

As the sheaves $\mathcal{L}_{n}^{q, 1}$ have vanishing cohomology on affine open subsets of $X$, and the transition maps $\mathcal{L}_{n+1}^{q, 1} \rightarrow \mathcal{L}_{n}^{q, 1}$ are surjective, the projective systems $\left(\mathcal{L}_{n}^{q, 1}\right)_{n \geqslant 1}$ are $\varliminf_{n}$-acyclic, and the canonical morphism

$$
\mathcal{L}^{\bullet} \rightarrow \underset{n}{\mathbb{\operatorname { l i m }}} \mathcal{L}_{n}^{\bullet, 1}
$$

is an isomorphism. This isomorphism provides a spectral sequence

$$
E_{2}^{i, j}=R^{i} \underset{n}{\lim _{n}} \mathcal{H}^{j}\left(\mathcal{L}_{n}^{\bullet, 1}\right) \Rightarrow \mathcal{H}^{i+j}\left(\mathcal{L}^{\bullet}\right)
$$

on which $F$ acts. As $F$ is nilpotent on the terms $E_{2}^{i, j}$ for $j<N$, it follows that $F$ is also nilpotent on $\mathcal{H}^{q}\left(\mathcal{L}^{\bullet}\right)$ for $q<N$. However, $F$ becomes an isomorphism after tensorization by $K$, so this implies assertion (i).

Thanks to Theorem 2.4, $R^{i} f_{q *}\left(W \mathcal{I}_{q}\right)_{K}=0$ for all $q$ and all $i \geqslant 1$. Therefore, the canonical morphism

$$
f_{\bullet *}\left(W \mathcal{I}_{\bullet}\right)_{K} \rightarrow \mathbb{R} f_{\bullet *}\left(W \mathcal{I}_{\bullet}\right)_{K}
$$

is an isomorphism. Then assertion (ii) follows from assertion (i). 


\section{WITT VECTOR COHOMOLOGY OF SINGULAR VARIETIES}

\section{Witt vector cohomology and rigid cohomology}

In this section, we first recall how to compute rigid cohomology in terms of de Rham complexes for the Zariski topology. Using this description, we construct the canonical morphism from rigid cohomology with compact supports to Witt vector cohomology with compact supports.

4.1 We begin by recalling the general construction of tubes [Ber96], which is sufficient to define the rigid cohomology groups of a proper $k$-scheme.

Let $X$ be a separated $k$-scheme of finite type, and let $X \hookrightarrow \mathbb{P}$ be a closed immersion into a smooth formal $W$-scheme. The formal scheme $\mathbb{P}$ has a generic fibre $\mathbb{P}_{K}$, which is a rigid analytic space, endowed with a continuous morphism sp $: \mathbb{P}_{K} \rightarrow \mathbb{P}$, the specialization morphism, such that $\mathrm{sp}^{-1}(\mathbb{U})=\mathbb{U}_{K}$ for any open subset $\mathbb{U} \subset \mathbb{P}$ and $\mathrm{sp}_{*} \mathcal{O}_{\mathbb{P}_{K}}=\mathcal{O}_{\mathbb{P}, K}$. When $\mathbb{P}$ is an affine formal scheme $\operatorname{Spf} A$, where $A$ is a $W$-algebra which is topologically of finite type, its generic fibre is the affinoid space $\operatorname{Spm} A_{K}$, defined by the Tate algebra $A_{K}$.

If $\mathbb{P}=\operatorname{Spf} A$, and if $f_{1}, \ldots, f_{r} \in A$ is a family of generators of the ideal $\mathcal{J}$ of $X$ in $\mathbb{P}$, the tube ]$X\left[\mathbb{P}\right.$ of $X$ in $\mathbb{P}_{K}$ is the admissible open subset defined by

$$
] X\left[_{\mathbb{P}}=\left\{x \in \mathbb{P}_{K}|\forall i,| f_{i}(x) \mid<1\right\},\right.
$$

where, for a point $x \in \mathbb{P}_{K}$ corresponding to a maximal ideal $\mathfrak{m} \subset A_{K},\left|f_{i}(x)\right|$ is the absolute value of the class of $f_{i}$ in the residue field $K(x)=A_{K} / \mathfrak{m}$. When $\mathbb{P}$ is not affine, one can choose a covering of $\mathbb{P}$ by open affine subsets $\mathbb{U}_{i}$, and the generic fibres $\mathbb{U}_{i, K}$ of the $\mathbb{U}_{i}$ provide an admissible covering of $\mathbb{P}_{K}$. Then the tube $] X[\mathbb{P}$ can be defined by gluing the tubes $] X \cap \mathbb{U}_{i}\left[\mathbb{U}_{i} \subset \mathbb{U}_{i, K}\right.$.

4.2 We introduce now the $\mathcal{O}_{\mathbb{P}}$-algebra $\mathcal{A}_{X, \mathbb{P}}$ of analytic functions on $] X{ }_{\mathbb{P}}$, the de Rham cohomology of which defines rigid cohomology.

The specialization morphism sp $: \mathbb{P}_{K} \rightarrow \mathbb{P}$ maps $] X[\mathbb{P}$ to $X$. Thus, one can define a sheaf of

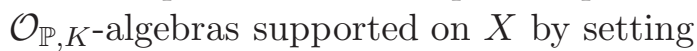

$$
\mathcal{A}_{X, \mathbb{P}}=\operatorname{sp}_{*} \mathcal{O}_{] X[\mathbb{P}}
$$

The differentiation of analytic functions on $] X\left[\mathbb{P}\right.$ endows $\mathcal{A}_{X, \mathbb{P}}$ with a canonical integrable connection, allowing one to define the de Rham complex $\mathcal{A}_{X, \mathbb{P}} \otimes \Omega_{\mathbb{P}}^{*}$. For any affine open subset $\mathbb{U} \subset \mathbb{P}$ and any $j$, we have $\Gamma\left(\mathbb{U}_{K}, \Omega_{\mathbb{P}_{K}}^{j}\right) \cong \Gamma\left(\mathbb{U}, \Omega_{\mathbb{P}}^{j}\right) \otimes K$. It follows that there is a canonical isomorphism of complexes

$$
\mathcal{A}_{X, \mathbb{P}} \otimes_{\mathcal{O}_{\mathbb{P}}} \Omega_{\mathbb{P}}^{\bullet} \cong \operatorname{sp}_{*}\left(\Omega_{j X[\mathbb{P}}^{\bullet}\right) .
$$

Moreover, the above description of $] X[\mathbb{P}$ in the affine case shows that the inverse image of an affine open subset $\mathbb{U} \subset \mathbb{P}$ is quasi-Stein in the sense of Kiehl [Kie67, Definition 2.3]. Therefore, Kiehl's vanishing theorem for coherent analytic sheaves implies that $R^{i} \operatorname{sp}_{*}\left(\Omega_{] X[\mathbb{P}}^{j}\right)=0$ for all $j$ and all $i \geqslant 1$, and we obtain

$$
\mathcal{A}_{X, \mathbb{P}} \otimes_{\mathcal{O}_{\mathbb{P}}} \Omega_{\mathbb{P}}^{\bullet} \cong \mathbb{R s p}_{*}\left(\Omega_{]_{[\mathbb{P}}}\right)
$$

in the derived category $D^{\mathrm{b}}(X, K)$.

If $X \hookrightarrow \mathbb{P}$ and $X \hookrightarrow \mathbb{P}^{\prime}$ are two closed immersions of $X$ into smooth formal $W$-schemes, there exists in $D^{\mathrm{b}}(X, K)$ a canonical isomorphism [Ber97a, Corollaire 1.5]

$$
\mathcal{A}_{X, \mathbb{P}} \otimes \Omega_{\mathbb{P}}^{\bullet} \cong \mathcal{A}_{X, \mathbb{P}^{\prime}} \otimes \Omega_{\mathbb{P}^{\prime}}^{\bullet}
$$

Thus, up to canonical isomorphism, the complex $\mathcal{A}_{X, \mathbb{P}} \otimes \Omega_{\mathbb{P}}^{\bullet}$ does not depend in $D^{\mathrm{b}}(X, K)$ on the choice of the embedding $X \hookrightarrow \mathbb{P}$. In particular, the de Rham cohomology of $] X[\mathbb{P}$ does not depend on this choice. When $X$ is a proper $k$-scheme and can be embedded in a smooth formal scheme $\mathbb{P}$ 


\section{P. Berthelot, S. Bloch and H. Esnault}

as above, its rigid cohomology is defined by

$$
\mathbb{R} \Gamma_{\text {rig }}(X / K)=\mathbb{R} \Gamma(] X\left[\mathbb{P}, \Omega_{]_{[\mathbb{P}}}\right) \cong \mathbb{R} \Gamma\left(X, \mathcal{A}_{X, \mathbb{P}} \otimes \Omega_{\mathbb{P}}^{\bullet}\right) .
$$

If such an embedding does not exist, one can choose a covering of $X$ by affine open subsets $X_{i}$, and, for each $i$, a closed immersion of $X_{i}$ in a smooth affine formal scheme $\mathbb{P}_{i}$ over $W$. Using the diagonal immersions for finite intersections $X_{i_{0}, \ldots, i_{r}}=X_{i_{0}} \cap \cdots \cap X_{i_{r}}$, and the corresponding algebras $\mathcal{A}_{X_{i_{0}, \ldots, i_{r}}, \mathbb{P}_{i_{0}} \times \cdots \times \mathbb{P}_{i_{r}}}$, one can build a Čech-de Rham double complex. When $X$ is proper, the cohomology of the associated total complex defines rigid cohomology.

4.3 The previous constructions can be extended as follows to define rigid cohomology with compact supports for separated $k$-schemes of finite type.

Let $U$ be such a scheme, $U \hookrightarrow X$ an open immersion of $U$ in a proper $k$-scheme $X, Y=X \backslash U$. Assume that there exists a closed immersion $X \hookrightarrow \mathbb{P}$ of $X$ into a smooth formal scheme $\mathbb{P}$, and denote by $u:] Y[\mathbb{P} \hookrightarrow] X[\mathbb{P}$ the inclusion of the tube of $Y$ into the tube of $X$. Then $] Y[\mathbb{P}$ is an admissible open subset of $] X[\mathbb{P}$, and, by construction [Ber86], the rigid cohomology with compact supports of $U$ is defined by

$$
\mathbb{R} \Gamma_{\text {rig,c }}(U / K)=\mathbb{R} \Gamma(] X\left[\mathbb{P},\left(\Omega_{]_{X \mathbb{P}}}^{\bullet} \rightarrow u_{*}\left(\Omega_{]_{Y[\mathbb{P}}}\right)\right)_{\mathrm{t}}\right),
$$

where the subscript $\mathrm{t}$ denotes the total complex associated to a double complex. Using the algebras $\mathcal{A}_{X, \mathbb{P}}$ and $\mathcal{A}_{Y, \mathbb{P}}$ defined in $\S 4.2$, we can rephrase this definition as

$$
\mathbb{R} \Gamma_{\text {rig }, \mathrm{c}}(U / K)=\mathbb{R} \Gamma\left(X,\left(\mathcal{A}_{X, \mathbb{P}} \otimes_{\mathcal{O}_{\mathbb{P}}} \Omega_{\mathbb{P}}^{\bullet} \rightarrow \mathcal{A}_{Y, \mathbb{P}} \otimes_{\mathcal{O}_{\mathbb{P}}} \Omega_{\mathbb{P}}^{\bullet}\right)_{\mathrm{t}}\right) .
$$

This cohomology only depends on $U$, and has the usual properties of cohomology with compact supports.

If there is no embedding of a compactification $X$ of $U$ in a smooth formal $W$-scheme, the rigid cohomology with compact supports of $U$ can still be defined using coverings by affine open subsets and Čech complexes as in $\S 4.2$.

Proposition 4.4. Let $\mathbb{P}$ be a smooth and separated formal scheme over $W$, with special fibre $P$, and let $X$ be a closed subscheme of $P$.

(i) The datum of a $\sigma$-semilinear lifting $F: \mathbb{P} \rightarrow \mathbb{P}$ of the absolute Frobenius endomorphism of $P$ defines a ring homomorphism

$$
\mathcal{A}_{X, \mathbb{P}} \rightarrow W \mathcal{O}_{X, K}
$$

functorial in $(X, \mathbb{P}, F)$.

(ii) Without assumption on the existence of $F$, there exists in $D^{\mathrm{b}}(X, K)$ a morphism

$$
a_{X, \mathbb{P}}: \mathcal{A}_{X, \mathbb{P}} \otimes_{\mathcal{O}_{\mathbb{P}}} \Omega_{\mathbb{P}}^{\bullet} \rightarrow W \mathcal{O}_{X, K},
$$

functorial in $(X, \mathbb{P})$, equal to the composed morphism

$$
\mathcal{A}_{X, \mathbb{P}} \otimes_{\mathcal{O}_{\mathbb{P}}} \Omega_{\mathbb{P}}^{\bullet} \rightarrow \mathcal{A}_{X, \mathbb{P}} \stackrel{(4.4)}{\longrightarrow} W \mathcal{O}_{X, K}
$$

whenever there exists a lifting of the Frobenius endomorphism on $\mathbb{P}$, and compatible with the canonical isomorphism (4.1)

$$
\mathcal{A}_{X, \mathbb{P}} \otimes_{\mathcal{O}_{\mathbb{P}}} \Omega_{\mathbb{P}}^{\bullet} \cong \mathcal{A}_{X, \mathbb{P}^{\prime}} \otimes_{\mathcal{O}_{\mathbb{P}}} \Omega_{\mathbb{P}^{\prime}}^{\bullet}
$$

for two closed immersions of $X$ into smooth and separated formal schemes $\mathbb{P}$ and $\mathbb{P}^{\prime}$.

(iii) Let $U$ be an open subset of $X, Y=X \backslash U$, and $\mathcal{I} \subset \mathcal{O}_{X}$ a coherent ideal such that $V(\mathcal{I})=Y$. Without assumption on the existence of $F$, there exists in $D^{\mathrm{b}}(X, K)$ a morphism

$$
a_{U, X, \mathbb{P}}:\left(\mathcal{A}_{X, \mathbb{P}} \otimes_{\mathcal{O}_{\mathbb{P}}} \Omega_{\mathbb{P}}^{\bullet} \rightarrow \mathcal{A}_{Y, \mathbb{P}} \otimes_{\mathcal{O}_{\mathbb{P}}} \Omega_{\mathbb{P}}^{\bullet}\right)_{\mathrm{t}} \rightarrow W \mathcal{I}_{K},
$$




\section{WiTT VECTOR COHOMOLOGY OF SINGULAR VARIETIES}

functorial with respect to morphisms $\left(U^{\prime}, X^{\prime}, \mathbb{P}^{\prime}\right) \rightarrow(U, X, \mathbb{P})$ such that $U^{\prime} \rightarrow U$ is proper, equal to (4.5) when $U=X$, and compatible with the canonical isomorphisms

$$
\left(\mathcal{A}_{X, \mathbb{P}} \otimes_{\mathcal{O}_{\mathbb{P}}} \Omega_{\mathbb{P}}^{\bullet} \rightarrow \mathcal{A}_{Y, \mathbb{P}} \otimes_{\mathcal{O}_{\mathbb{P}}} \Omega_{\mathbb{P}}^{\bullet}\right)_{t} \cong\left(\mathcal{A}_{X, \mathbb{P}^{\prime}} \otimes_{\mathcal{O}_{\mathbb{P}}} \Omega_{\mathbb{P}^{\prime}}^{\bullet} \rightarrow \mathcal{A}_{Y, \mathbb{P}^{\prime}} \otimes_{\mathcal{O}_{\mathbb{P}}} \Omega_{\mathbb{P}^{\prime}}\right)_{\mathrm{t}}
$$

for two closed immersions of $X$ into smooth formal schemes $\mathbb{P}$ and $\mathbb{P}^{\prime}$.

Proof. Let $\mathcal{J} \subset \mathcal{O}_{\mathbb{P}}$ be the ideal defining $X, \mathcal{P}(\mathcal{J})$ the divided power envelope of $\mathcal{J}$, with compatibility with the natural divided powers of $p$, and $\widehat{\mathcal{P}}(\mathcal{J})$ its $p$-adic completion. We recall first from [Ber97a, p. 342] that there exists a functorial homomorphism of $\mathcal{O}_{\mathbb{P}}$-algebras

$$
\mathcal{A}_{X, \mathbb{P}} \rightarrow \widehat{\mathcal{P}}(\mathcal{J})_{K}
$$

Let $P_{n}$ be the reduction of $\mathbb{P} \bmod p^{n}$, and let $\mathcal{J}_{n}=\mathcal{J O}_{P_{n}}$ be the ideal of $X$ in $P_{n}$. Thanks to the compatibility condition with the divided powers of $p$, there is a canonical isomorphism

$$
\widehat{\mathcal{P}}(\mathcal{J}) / p^{n} \widehat{\mathcal{P}}(\mathcal{J}) \stackrel{\sim}{\longrightarrow} \mathcal{P}\left(\mathcal{J}_{n}\right),
$$

where $\mathcal{P}\left(\mathcal{J}_{n}\right)$ is the divided power envelope of $\mathcal{J}_{n}$ with the same compatibility condition, and we obtain

$$
\widehat{\mathcal{P}}(\mathcal{J}) \stackrel{\sim}{\longrightarrow} \underset{n}{\lim } \mathcal{P}\left(\mathcal{J}_{n}\right)
$$

Thus, it suffices to define a compatible family of functorial ring homomorphisms

$$
\mathcal{P}\left(\mathcal{J}_{n}\right) \rightarrow W_{n} \mathcal{O}_{X}
$$

to obtain a morphism (4.4).

Let us assume that $\mathbb{P}$ is endowed with a lifting $F$ of the Frobenius morphism. As $\mathcal{O}_{\mathbb{P}}$ is $p$-torsion free, the homomorphism $F: \mathcal{O}_{\mathbb{P}} \rightarrow \mathcal{O}_{\mathbb{P}}$ defines a section $s_{F}: \mathcal{O}_{\mathbb{P}} \rightarrow W \mathcal{O}_{\mathbb{P}}$ of the reduction homomorphism $W \mathcal{O}_{\mathbb{P}} \rightarrow \mathcal{O}_{\mathbb{P}}$, characterized by the fact that $w_{i}\left(s_{F}(x)\right)=F^{i}(x)$ for any $x \in \mathcal{O}_{\mathbb{P}}$ and any ghost component $w_{i}$ (see [Ill79, $\left.0, \S 1.3\right]$ ). Composing $s_{F}$ with the homomorphisms $W \mathcal{O}_{\mathbb{P}} \rightarrow W \mathcal{O}_{P} \rightarrow W_{n} \mathcal{O}_{P}$ and factorizing, we obtain for all $n \geqslant 1$ a homomorphism

$$
\mathcal{O}_{P_{n}} \rightarrow W_{n} \mathcal{O}_{P} \rightarrow W_{n} \mathcal{O}_{X}
$$

which maps $\mathcal{J}_{n}$ to $V W_{n-1} \mathcal{O}_{X} \subset W_{n} \mathcal{O}_{X}$. The ideal $V W_{n-1} \mathcal{O}_{X}$ has a natural structure of divided power ideal (compatible with the divided powers of $p$ ), defined by $(V x)^{[i]}=\left(p^{i-1} / i !\right) V\left(x^{i}\right)$ for all $i \geqslant 1$. Therefore, this homomorphism factors through a homomorphism $\mathcal{P}\left(\mathcal{J}_{n}\right) \rightarrow W_{n} \mathcal{O}_{X}$. This provides the compatible family of homomorphisms defining (4.4), and it is clear that the homomorphism obtained in this way is functorial in $(X, \mathbb{P}, F)$.

By composition with the augmentation morphism $\mathcal{A}_{X, \mathbb{P}} \otimes_{\mathcal{O}_{\mathbb{P}}} \Omega_{\mathbb{P}}^{\bullet} \rightarrow \mathcal{A}_{X, \mathbb{P}}$, we obtain a morphism of complexes

$$
a_{X, \mathbb{P}, F}: \mathcal{A}_{X, \mathbb{P}} \otimes_{\mathcal{O}_{\mathbb{P}}} \Omega_{\mathbb{P}}^{\bullet} \rightarrow W \mathcal{O}_{X, K},
$$

which is still functorial in $(X, \mathbb{P}, F)$. If $X \hookrightarrow \mathbb{P}$ and $X \hookrightarrow \mathbb{P}^{\prime}$ are two closed immersions in smooth formal schemes endowed with liftings of Frobenius $F$ and $F^{\prime}$, we can endow $\mathbb{P}^{\prime \prime}=\mathbb{P} \times_{W} \mathbb{P}^{\prime}$ with $F \times F^{\prime}$, and embed $X$ diagonally into $\mathbb{P}^{\prime \prime}$. Applying the functoriality of this construction to the two projections, we obtain a commutative diagram

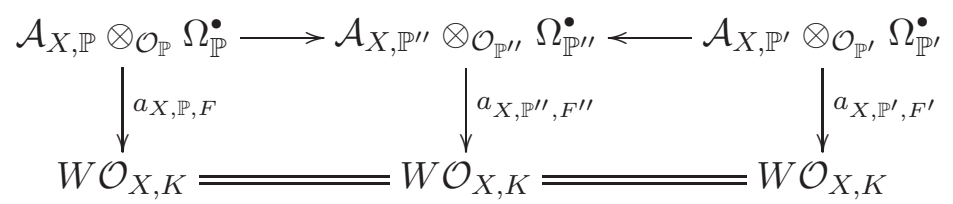

in which the morphisms of the top row are quasi-isomorphisms. In $D^{\mathrm{b}}(X, K)$, the composed isomorphism $\mathcal{A}_{X, \mathbb{P}} \otimes_{\mathcal{O}_{\mathbb{P}}} \Omega_{\mathbb{P}}^{\bullet} \cong \mathcal{A}_{X, \mathbb{P}^{\prime}} \otimes_{\mathcal{O}_{\mathbb{P}^{\prime}}} \Omega_{\mathbb{P}^{\prime}}^{\bullet}$ is the canonical isomorphism (4.1) (see [Ber97a, Corollaire 1.5]), which shows the compatibility asserted in assertion (ii). In the particular case where $\mathbb{P}^{\prime}=\mathbb{P}$, this 


\section{P. Berthelot, S. Bloch and H. Esnault}

composed isomorphism is the identity, and we obtain that, as a morphism of $D^{\mathrm{b}}(X, K)$, the morphism $a_{X, \mathbb{P}, F}$ does not depend on the choice of $F$. A similar argument shows that, in $D^{\mathrm{b}}(X, K)$, it is functorial with respect to morphisms $\left(X^{\prime}, \mathbb{P}^{\prime}\right) \rightarrow(X, \mathbb{P})$ without compatibility with Frobenius liftings. Thus, if we define $a_{X, \mathbb{P}}$ to be the image in $D^{\mathrm{b}}(X, K)$ of $a_{X, \mathbb{P}, F}$, assertion (ii) is true when there exists a lifting of Frobenius on $\mathbb{P}$.

In the general case, we can choose an affine covering $\mathbb{P}_{i}$ of $\mathbb{P}$, and a lifting of Frobenius $F_{i}$ on each $\mathbb{P}_{i}$. Then, if $X_{i_{0}, \ldots, i_{n}}=X \cap \mathbb{P}_{i_{0}} \cap \cdots \cap \mathbb{P}_{i_{n}}$ and $j_{i_{0}, \ldots, i_{n}}$ denotes its inclusion in $X$, the complex $\mathcal{A}_{X, \mathbb{P}} \otimes_{\mathcal{O}_{\mathbb{P}}} \Omega_{\mathbb{P}}^{\bullet}$ is quasi-isomorphic to the total complex associated to the double complex

$$
\prod_{i} j_{i *}\left(\mathcal{A}_{X_{i}, \mathbb{P}_{i}} \otimes \Omega_{\mathbb{P}_{i}}^{\bullet}\right) \rightarrow \cdots \rightarrow \prod_{i_{0}, \ldots, i_{n}} j_{i_{0}, \ldots, i_{n} *}\left(\mathcal{A}_{X_{i_{0}, \ldots, i_{n}}, \mathbb{P}_{i_{0}} \times \cdots \times \mathbb{P}_{i_{n}}} \otimes \Omega_{\mathbb{P}_{i_{0}} \times \cdots \times \mathbb{P}_{i_{n}}}^{\cdot}\right) \rightarrow \cdots,
$$

while $W \mathcal{O}_{X, K}$ is quasi-isomorphic to the Čech resolution

$$
\prod_{i} j_{i *}\left(W \mathcal{O}_{X_{i}, K}\right) \rightarrow \cdots \rightarrow \prod_{i_{0}, \ldots, i_{n}} j_{i_{0}, \ldots, i_{n} *}\left(W \mathcal{O}_{X_{i_{0}, \ldots, i_{n}}, K}\right) \rightarrow \cdots
$$

Then we can define $a_{X, \mathbb{P}}$ as the image in $D^{\mathrm{b}}(X, K)$ of the morphism defined by the collection of all $a_{X_{i_{0}, \ldots, i_{n}}, \mathbb{P}_{i_{0}} \times \cdots \times \mathbb{P}_{i_{n}}, F_{i_{0}} \times \cdots \times F_{i_{n}}}$, and assertion (ii) is verified as above.

Let $U$ be an open subset of $X$, and assume again that there exists a lifting $F$ of the Frobenius endomorphism on $\mathbb{P}$. Then the previous constructions can be applied both to $X$ and to $Y=X \backslash U$. By functoriality, they provide a morphism of complexes

$$
\left(\mathcal{A}_{X, \mathbb{P}} \otimes_{\mathcal{O}_{\mathbb{P}}} \Omega_{\mathbb{P}}^{\bullet} \rightarrow \mathcal{A}_{Y, \mathbb{P}} \otimes_{\mathcal{O}_{\mathbb{P}}} \Omega_{\mathbb{P}}^{\bullet}\right)_{t} \rightarrow\left(W \mathcal{O}_{X, K} \rightarrow W \mathcal{O}_{Y, K}\right)
$$

As the length 1 complex $W \mathcal{O}_{X, K} \rightarrow W \mathcal{O}_{Y, K}$ is a resolution of $W \mathcal{I}_{K}$, we can define $a_{U, X, \mathbb{P}}$ as the image of this morphism in $D^{\mathrm{b}}(X, K)$. If $Y=\emptyset$, then we simply obtain (4.5). To check the functoriality, we may assume that $U^{\prime}$ is dense in $X^{\prime}$, because $W \mathcal{I}_{K}^{\prime}$ does not change if we replace $X^{\prime}$ by the closure of $U^{\prime}$ in $X^{\prime}$, by Theorem 2.4 , and similarly $\left(\mathcal{A}_{X, \mathbb{P}} \otimes_{\mathcal{O}_{\mathbb{P}}} \Omega_{\mathbb{P}}^{\bullet} \rightarrow \mathcal{A}_{Y, \mathbb{P}} \otimes_{\mathcal{O}_{\mathbb{P}}} \Omega_{\mathbb{P}}^{\bullet}\right)_{\mathrm{t}}$ does not change up to canonical isomorphism in $D^{\mathrm{b}}(X, K)$, by the basic properties of rigid cohomology. Then the properness of $U^{\prime} \rightarrow U$ allows us to assume that $Y^{\prime}=X^{\prime} \backslash U^{\prime}$ is defined by $\mathcal{I}^{\prime}=\mathcal{I} \mathcal{O}_{X^{\prime}}$, and the functoriality is clear. Finally, the compatibility with the canonical isomorphism for two embeddings of $X$ into smooth formal schemes results from the same assertion for the morphisms (4.5) relative to $X$ and $Y$.

When $F$ cannot be lifted to $\mathbb{P}$, one can proceed with Čech coverings as in assertion (ii) to define (4.6), and the same properties hold.

Remark. If $X$ is quasi-projective, one can always find a closed immersion of $X$ into a smooth formal scheme $\mathbb{P}$ endowed with a lifting of Frobenius $F$, since it suffices to choose for $\mathbb{P}$ an open subscheme of a projective space, endowed with the endomorphism induced by some lifting of the Frobenius endomorphism of the projective space.

TheOREm 4.5. Let $U$ be a separated $k$-scheme of finite type. In $D^{\mathrm{b}}(K)$, there exists a functorial morphism

$$
a_{U}: \mathbb{R} \Gamma_{\text {rig, },}(U / K) \rightarrow \mathbb{R} \Gamma_{\mathrm{c}}\left(U, W \mathcal{O}_{U, K}\right),
$$

equal, for a proper and smooth $k$-scheme $X$, to the canonical morphism

$$
\begin{aligned}
\mathbb{R} \Gamma_{\text {rig }}(X / K) \stackrel{\sim}{\longrightarrow} \mathbb{R} \Gamma_{\text {crys }}(X / W)_{K} \stackrel{\sim}{\longrightarrow} & \mathbb{R} \Gamma\left(X, W \Omega_{X}^{\bullet}\right)_{K} \\
& \stackrel{\mathbb{R} \Gamma\left(X, W \mathcal{O}_{X}\right)_{K}}{ }
\end{aligned}
$$

In (4.13), the first isomorphism is the comparison isomorphism between rigid and crystalline cohomologies [Ber97a, Proposition 1.9], the second is the comparison isomorphism between crystalline 


\section{WiTT VECTOR COHOMOLOGY OF SINGULAR VARIETIES}

and de Rham-Witt cohomologies [Ill79, II, equation (1.3.2)], and the third map is defined by the augmentation morphism of the de Rham-Witt complex.

Proof. Let $U \hookrightarrow X$ be an open immersion of $U$ in a proper $k$-scheme. Let us assume first that there exists a closed immersion $X \hookrightarrow \mathbb{P}$ of $X$ into a smooth formal scheme $\mathbb{P}$. To define $a_{U}$, we apply the functor $\mathbb{R} \Gamma(X,-)$ to the morphism $a_{U, X, \mathbb{P}}$ defined in (4.6). Owing to the compatibility property of Proposition 4.4(iii), we obtain in this way a morphism of $D^{\mathrm{b}}(K)$ which does not depend, up to canonical isomorphism, on the choice of $\mathbb{P}$. Using the functoriality of $a_{U, X, \mathbb{P}}$, it is easy to check that it does not depend either on the choice of the compactification $X$ of $U$, and that it is functorial with respect to $U$.

When $U=X$ is a proper and smooth $k$-scheme, and $X$ is embeddable in a smooth formal scheme $\mathbb{P}$ with a lifting $F$ of the Frobenius endomorphism, the homomorphisms (4.8) used to construct $a_{X, \mathbb{P}, F}$ are the homomorphisms used in [Ill79, II, equation (1.1.5)] to construct the morphism of complexes $\widehat{\mathcal{P}}(\mathcal{J}) \otimes \Omega_{\mathbb{P}}^{\bullet} \rightarrow W \Omega_{X}^{\bullet}$ which defines the isomorphism between crystalline and de Rham-Witt cohomologies. Therefore, the comparison isomorphisms which appear in (4.13) sit in a commutative diagram

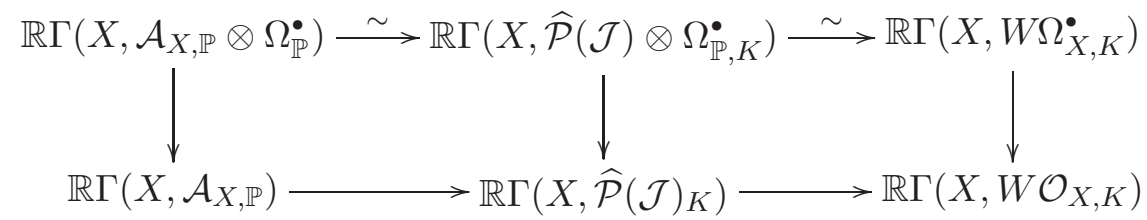

in which the vertical maps are induced by the augmentation morphisms of the complexes appearing in the upper row. The equality of the morphisms (4.12) and (4.13) follows immediately.

In the general case where there is no smooth embedding $\mathbb{P}$ of $X$, or no Frobenius lifting on $\mathbb{P}$, one can again use a covering of $X$ by affine open subsets $X_{i}$, and closed immersions $X_{i} \hookrightarrow \mathbb{P}_{i}$ in smooth formal schemes $\mathbb{P}_{i}$ endowed with liftings of Frobenius, to construct Čech complexes as in (4.10) and (4.11). One can then use on each intersection of the covering the corresponding morphism (4.6) and define in this way a morphism between the two Čech complexes. Applying $\mathbb{R} \Gamma(X,-)$, one gets in $D^{\mathrm{b}}(K)$ the morphism $a_{U}$. In the proper and smooth case, the fact that each map in the above diagram can be defined through Čech complexes shows that $a_{U}$ is still equal to (4.13).

The following verifications are left as an exercise for the reader.

Proposition 4.6. The morphism $a_{U}$ defined by Theorem 4.5 satisfies the following compatibility properties.

(i) The diagram

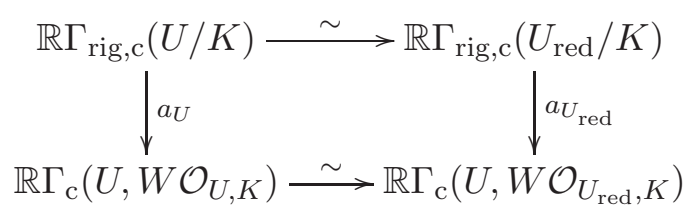

is commutative.

(ii) If $V \subset U$ is an open subset, and $T=U \backslash V$, the morphisms $a_{V}, a_{U}$, and $a_{T}$ define the following morphism of exact triangles.

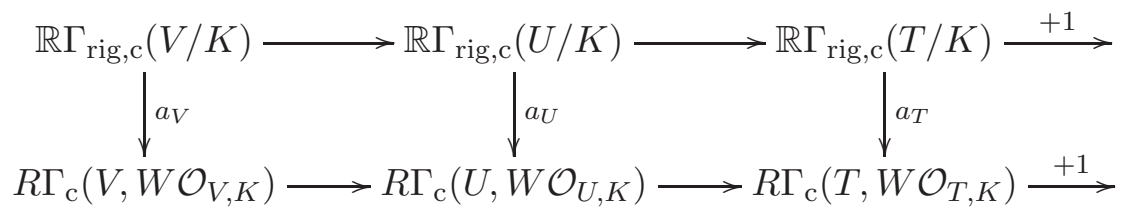




\section{P. Berthelot, S. Bloch and H. Esnault}

\section{Proof of the main theorem}

We prove here that the morphism $a_{U}$ constructed in Theorem 4.5 yields an identification of the slope $<1$ subspace of rigid cohomology with compact supports with Witt vector cohomology with compact supports, thus completing the proof of Theorem 1.1.

The next lemma allows us to use descent techniques to study the morphism $a_{U}$. We follow here the method of Chiarellotto and Tsuzuki to construct embeddings of a simplicial scheme in a smooth simplicial formal scheme (cf. [CT03, § 11.2] and [Tsu04, § 7.3]).

Lemma 5.1. Let $X$ be a separated reduced $k$-scheme of finite type, and let $X \hookrightarrow \mathbb{P}$ be a closed immersion into a smooth formal $W$-scheme endowed with a Frobenius lifting $F$. Let $N \in \mathbb{N}$ be a fixed integer. There exists a proper hypercovering $X$. of $X$, a $\mathbb{P}$-augmented simplicial formal $W$-scheme $\mathbb{P}$, endowed with a $\sigma$-semilinear endomorphism $F$. lifting the absolute Frobenius endomorphism of its special fibre, and a morphism of simplicial schemes $X \bullet \rightarrow \mathbb{P}$. above $X \hookrightarrow \mathbb{P}$, such that the following conditions are satisfied.

(i) For all $n$, the morphism $X_{n} \rightarrow \mathbb{P}_{n}$ is a closed immersion, and the projection $\mathbb{P}_{n} \rightarrow \mathbb{P}$ commutes with the Frobenius liftings.

(ii) The $N$-truncated simplicial scheme $\operatorname{sk}_{N}^{X}\left(X_{\bullet}\right)$ is an $N$-truncated simplicial resolution of $X$.

(iii) The canonical morphisms $X_{\bullet} \rightarrow \operatorname{cosk}_{N}^{X}\left(\operatorname{sk}_{N}^{X}\left(X_{\bullet}\right)\right)$ and $\mathbb{P} \bullet \rightarrow \operatorname{cosk}_{N}^{\mathbb{P}}\left(\operatorname{sk}_{N}^{\mathbb{P}}\left(\mathbb{P}_{\bullet}\right)\right)$ are isomorphisms.

(iv) For all $n, \mathbb{P}_{n}$ is smooth over $\mathbb{P}$, and the canonical morphism $\mathbb{P}_{n} \rightarrow \operatorname{cosk}_{n-1}^{\mathbb{P}}\left(\operatorname{sk}_{n-1}^{\mathbb{P}}\left(\mathbb{P}_{\bullet}\right)\right)_{n}$ is smooth.

Proof. Using de Jong's theorem, we can find an $N$-truncated simplicial resolution of $X$ (cf. Definition 3.1). We define $X_{\bullet}$ as the $N$-coskeleton over $X$ of this $N$-truncated resolution. Thus, $X_{\bullet}$ is a proper hypercovering of $X, \operatorname{sk}_{N}^{X}\left(X_{\bullet}\right)$ is our initial $N$-truncated simplicial resolution of $X$, and the morphism $X_{\bullet} \rightarrow \operatorname{cosk}_{N}^{X}\left(\operatorname{sk}_{N}^{X}\left(X_{\bullet}\right)\right)$ is an isomorphism.

For $n \in \mathbb{N}$, let $[n]$ denote the ordered set $\{0, \ldots, n\}$. Let $\Delta$ be the category which has the sets $[n]$ as objects, and the set of non-decreasing maps $[m] \rightarrow[n]$ as the set of morphisms from $[m]$ to $[n]$. Since the $X_{m}$ are quasi-projective over $k$, we can choose for each $m \leqslant N$ a closed immersion $i_{m}: X_{m} \hookrightarrow \mathbb{W}_{m}$, where $\mathbb{W}_{m}$ is a smooth formal $W$-scheme endowed with a lifting of Frobenius $F_{m}$. From $\mathbb{W}_{m}$, we construct a simplicial complex of smooth formal $W$-schemes $\Gamma_{n}\left(\mathbb{W}_{m}\right)$ by setting for all $n \geqslant 0$

$$
\Gamma_{n}\left(\mathbb{W}_{m}\right)=\prod_{\gamma:[m] \rightarrow[n]} \mathbb{W}_{m, \gamma}
$$

where the product is taken over all morphisms $\gamma:[m] \rightarrow[n]$ in $\Delta$, and $\mathbb{W}_{m, \gamma}=\mathbb{W}_{m}$ for all $\gamma$. If $\eta:\left[n^{\prime}\right] \rightarrow[n]$ is a morphism in $\Delta$, the corresponding morphism $\Gamma_{n}\left(\mathbb{W}_{m}\right) \rightarrow \Gamma_{n^{\prime}}\left(\mathbb{W}_{m}\right)$ is such that, for any $\gamma^{\prime}:[m] \rightarrow\left[n^{\prime}\right]$ in $\Delta$, its composition with the projection $\Gamma_{n^{\prime}}\left(\mathbb{W}_{m}\right) \rightarrow \mathbb{W}_{m, \gamma^{\prime}}=\mathbb{W}_{m}$ is the projection of $\Gamma_{n}\left(\mathbb{W}_{m}\right)$ on $\mathbb{W}_{m, \eta \circ \gamma^{\prime}}=\mathbb{W}_{m}$. We can then define a Frobenius lifting on the simplicial formal scheme $\Gamma_{\bullet}\left(\mathbb{W}_{m}\right)$ as being the product morphism defined by $F_{m}$ on each $\Gamma_{n}\left(\mathbb{W}_{m}\right)$; we still denote it by $F_{m}$.

The immersion $i_{m}$ defines a morphism of simplicial schemes $X_{\bullet} \rightarrow \Gamma_{\bullet}\left(\mathbb{W}_{m}\right)$ as follows: for each $n \geqslant 0$, the composition of $X_{n} \rightarrow \Gamma_{n}\left(\mathbb{W}_{m}\right)$ with the projection of index $\gamma$ is the morphism $X_{n} \rightarrow X_{m} \hookrightarrow \mathbb{W}_{m}$, where the first morphism is the morphism of $X$. defined by $\gamma$. We can now define a simplicial formal $W$-scheme $\mathbb{P}$. augmented towards $\mathbb{P}$ by setting

$$
\mathbb{P}_{\bullet}=\mathbb{P} \times \operatorname{Spf}(W) \prod_{0 \leqslant m \leqslant N} \Gamma \cdot\left(\mathbb{W}_{m}\right) .
$$

We define a morphism of simplicial schemes $X_{\bullet} \rightarrow \mathbb{P} \bullet$ using the composed morphism $X_{\bullet} \rightarrow X \rightarrow \mathbb{P}$ and the family of morphisms $X_{\bullet} \rightarrow \Gamma_{\bullet}\left(\mathbb{W}_{m}\right)$ defined above. By construction, this morphism is 


\section{WiTT VECTOR COHOMOLOGY OF SINGULAR VARIETIES}

compatible with the immersion $X \hookrightarrow \mathbb{P}$ via the augmentation morphisms. If we endow $\mathbb{P}$. with the Frobenius lifting $F \times \prod_{m} F_{m}$, then the augmentation $\mathbb{P} \bullet \rightarrow \mathbb{P}$ commutes with the Frobenius liftings.

To check the remaining properties, we observe first that $\mathbb{W} \stackrel{\sim}{\sim} \operatorname{cosk}_{N}^{\mathbb{P}}\left(\operatorname{sk}_{N}^{\mathbb{P}}\left(\mathbb{W}_{\bullet}\right)\right)$ thanks to [CT03, 11.2.5]. For each $m \leqslant N$, the morphism $X_{m} \rightarrow \Gamma_{m}\left(\mathbb{W}_{m}\right)$ is a closed immersion since the chosen immersion $X_{m} \hookrightarrow \mathbb{W}_{m}$ is one of the factors. Therefore, $X_{n} \rightarrow \mathbb{P}_{n}$ is a closed immersion for all $n \leqslant N$. Then, thanks to the previous property, it follows from the construction of coskeletons that $X_{n} \rightarrow \mathbb{P}_{n}$ is a closed immersion for all $n$. Finally, each $\mathbb{P}_{n}$ is smooth over $\mathbb{P}$ by construction, and the fact that $\mathbb{P}_{n}$ is smooth over $\left(\operatorname{cosk}_{n-1}^{\mathbb{P}}\left(\operatorname{sk}_{n-1}^{\mathbb{P}}\left(\mathbb{P}_{\bullet}\right)\right)\right)_{n}$ for all $n$ follows from [Tsu04, Lemma 7.3.3].

5.2 Proof of Theorem 1.1. We first recall the definition of the subspaces with prescribed slopes. Let $\bar{k}$ be an algebraic closure of $k$, and let $\bar{K}_{0}=\operatorname{Frac}(W(\bar{k}))$. If $E$ is a finite-dimensional $K$-vector space endowed with a $\sigma$-semilinear automorphism $F$, let $\bar{E}=\bar{K}_{0} \otimes_{K} E$ and $\bar{F}=\sigma_{\bar{K}_{0}} \otimes F$. The Dieudonné-Manin theorem provides for each $\lambda \in \mathbb{Q}$ a maximal $\bar{F}$-stable subspace $\bar{E}^{\lambda}$ on which $\bar{F}$ has purely slope $\lambda$, and asserts that $\bigoplus_{\lambda} \bar{E}^{\lambda} \cong E$. As these subspaces are invariant under the action of $\operatorname{Gal}(\bar{k} / k)$, there exists a unique $F$-stable subspace $E^{\lambda} \subset E$ such that $\bar{K}_{0} \otimes_{K} E^{\lambda} \stackrel{\sim}{\longrightarrow} \bar{E}^{\lambda}$ and $\bigoplus_{\lambda} E^{\lambda} \cong E$. The subspace $E^{\lambda}$ is the slope $\lambda$ subspace of $E$, and, for any $\rho \in \mathbb{R}$, we define the slope $<\rho$ subspace of $E$ by

$$
E^{<\rho}=\bigoplus_{\lambda<\rho} E^{\lambda}
$$

These spaces are functorial with respect to $(E, F)$, and define exact functors.

Let $X$ be a separated $k$-scheme of finite type. The morphism (4.12) induces canonical homomorphisms

$$
a_{X}^{q}: H_{\mathrm{rig}, \mathrm{c}}^{q}(X / K) \rightarrow H_{\mathrm{c}}^{q}\left(X, W \mathcal{O}_{X, K}\right)
$$

between the cohomology spaces. By functoriality, $a_{X}^{q}$ commutes to the Frobenius actions on both sides. The spaces $H_{\text {rig,c }}^{q}(X / K)$ are finite dimensional [Ber97a, Remarque 3.9 (i)], and their Frobenius endomorphism is an automorphism [EL93, Proposition 2.1]. Therefore, they have a slope decomposition, and $a_{X}^{q}$ induces a homomorphism

$$
b_{X}^{q}: H_{\text {rig }, \mathrm{c}}^{q}(X / K)^{<1} \rightarrow H_{\mathrm{c}}^{q}\left(X, W \mathcal{O}_{X, K}\right)
$$

which is the homomorphism (1.3) of Theorem 1.1.

To prove that $b_{X}^{q}$ is an isomorphism, we first observe that this is true when $X$ is proper and smooth over $k$. Indeed, $a_{X}^{q}$ is then induced by the morphism (4.13), and the isomorphisms which enter in the definition of (4.13) are compatible with Frobenius, hence induce isomorphisms on the slope $<1$ subspaces of the cohomology spaces. The theorem is then a consequence of the slope decomposition for de Rham-Witt cohomology [Ill79, II, equation (3.5.3)].

In the general case, we prove Theorem 1.1 by induction on $\operatorname{dim}(X)$. The diagram (4.14) allows us to assume that $X$ is reduced. In particular, the theorem holds for $\operatorname{dim}(X)=0$ thanks to the previous remark. Let us assume that all $b_{X}^{q}$ are isomorphisms when $\operatorname{dim}(X)<d$. If $U \subset X$ is an open subset of dimension $d$ of a proper and smooth $k$-scheme $X$, its closure $\bar{U}$ is proper and smooth over $k$, and $\operatorname{dim}(\bar{U} \backslash U)<d$. As all $b_{\bar{U}}^{q}$ are isomorphisms, the induction hypothesis and the commutativity of (4.15) imply that all $b_{U}^{q}$ are isomorphisms. Thus, the theorem also holds for any open subset of dimension $d$ of a proper and smooth $k$-scheme.

Let $X$ be an arbitrary reduced and separated $k$-scheme of dimension $d$. Using again (4.15) and the induction hypothesis, we may replace $X$ by an arbitrarily small neighbourhood $U$ of the generic points of its irreducible components. As the theorem holds for a scheme if and only if it holds for each of its connected components, we may shrink even more and assume that $U$ is irreducible and affine. 


\section{P. Berthelot, S. Bloch and H. Esnault}

Now let $U \hookrightarrow X$ be an open immersion of $U$ in a projective $k$-scheme $X$, such that $U$ is dense in $X$. Using again the same argument, it suffices to prove the theorem for $X$.

Since $X$ is projective, we can choose a closed immersion of $X$ into a smooth formal $W$-scheme $\mathbb{P}$ endowed with a Frobenius lifting $F$. Let $N$ be an integer such that $N \geqslant 2 d$. Then we can choose a proper hypercovering $X$. of $X$, a $\mathbb{P}$-augmented simplicial formal scheme $\mathbb{P}$. endowed with a Frobenius lifting $F_{\bullet}$, and a closed immersion of augmented simplicial schemes $X_{\bullet} \hookrightarrow \mathbb{P} \bullet$ above $X \hookrightarrow \mathbb{P}$, so that the conditions of Lemma 5.1 are satisfied.

In particular, there is a dense open subset $U \subset X$ such that the restriction $U$. of $X$. above $U$ is an étale hypercovering of $U$, and it suffices to prove the theorem for $U$. Let $Y=X \backslash U$. Since each $\mathbb{P}_{n}$ is smooth over $W$, the complexes $\left(\mathcal{A}_{X_{n}, \mathbb{P}_{n}} \otimes \Omega_{\mathbb{P}_{n}}^{\bullet} \rightarrow \mathcal{A}_{Y_{n}, \mathbb{P}_{n}} \otimes \Omega_{\mathbb{P}_{n}}\right)_{\mathrm{t}}$ are defined. By functoriality, they define a complex of sheaves on $X$. (see $[\operatorname{Del} 74, \S 5.1 .6]$ ), and we set

$$
H_{\text {rig,c }}^{*}\left(U_{\bullet} / K\right):=H^{*}\left(X_{\bullet},\left(\mathcal{A}_{X_{\bullet}, \mathbb{P} \bullet} \otimes \Omega_{\mathbb{P}_{\bullet}}^{\bullet} \rightarrow \mathcal{A}_{Y_{\bullet}, \mathbb{P} \bullet} \otimes \Omega_{\mathbb{P}_{\bullet}}\right)_{t}\right) .
$$

As usual, there is a biregular spectral sequence relating the cohomology of the individual complexes on the $X_{n}$ to the cohomology of the global complex on $X_{\bullet}$, which can be written here as

$$
E_{1}^{i, j}=H_{\mathrm{rig}, \mathrm{c}}^{j}\left(U_{i} / K\right) \Rightarrow H_{\mathrm{rig}, \mathrm{c}}^{i+j}\left(U_{\bullet} / K\right) .
$$

By functoriality, this spectral sequence is endowed with a Frobenius automorphism. Moreover, the $E_{1}^{i, j}$ terms are finite-dimensional $K$-vector spaces, so the spaces $H_{\text {rig,c }}^{i+j}\left(U_{\bullet} / K\right)$ are also finite dimensional. Since the subspace of slope $<1$ is an exact functor on the category of finite-dimensional $K$-vector spaces endowed with a $\sigma$-semilinear automorphism, we obtain a biregular spectral sequence

$$
E_{1}^{i, j}=H_{\text {rig,c }}^{j}\left(U_{i} / K\right)^{<1} \Rightarrow H_{\text {rig,c }}^{i+j}\left(U_{\bullet} / K\right)^{<1} .
$$

Let $\mathcal{I} \subset \mathcal{O}_{X}$ be a coherent ideal such that $V(\mathcal{I})=Y$, and set $\mathcal{I}_{n}=\mathcal{I O}_{X_{n}}, Y_{n}=V\left(\mathcal{I}_{n}\right) \subset X_{n}$. The sheaves $W \mathcal{O}_{X_{n}}, W \mathcal{O}_{Y_{n}}$, and $W \mathcal{I}_{n}$ define sheaves $W \mathcal{O}_{X_{\bullet}}, W \mathcal{O}_{Y_{\bullet}}$, and $W \mathcal{I}_{\bullet}$ on $X_{\bullet}$, with an exact sequence

We define

$$
0 \rightarrow W \mathcal{I}_{\bullet} \rightarrow W \mathcal{O}_{X} \rightarrow W \mathcal{O}_{Y_{\bullet}} \rightarrow 0
$$

$$
H_{\mathrm{c}}^{*}\left(U_{\bullet}, W \mathcal{O}_{U_{\bullet}, K}\right)=H^{*}\left(X_{\bullet}, W \mathcal{I}_{\bullet}, K\right)
$$

and we obtain a biregular spectral sequence

$$
E_{1}^{i, j}=H_{\mathrm{c}}^{j}\left(U_{i}, W \mathcal{O}_{U_{i}, K}\right) \Rightarrow H_{\mathrm{c}}^{i+j}\left(U_{\bullet}, W \mathcal{O}_{U_{\bullet}, K}\right) .
$$

As $F$ is an endomorphism of the simplicial scheme $\mathbb{P}_{\bullet}$, the homomorphisms (4.4) define morphisms of sheaves of rings $\mathcal{A}_{X_{\bullet}, \mathbb{P} \bullet} \rightarrow W \mathcal{O}_{X_{\bullet}, K}$ and $\mathcal{A}_{Y_{\bullet}, \mathbb{P}_{\bullet}} \rightarrow W \mathcal{O}_{Y_{\bullet}, K}$ on $X$, from which we derive a morphism of complexes

$$
\left(\mathcal{A}_{X_{\bullet}, \mathbb{P} \bullet} \otimes \Omega_{\mathbb{P}_{\bullet}} \rightarrow \mathcal{A}_{Y_{\bullet}, \mathbb{P}} \otimes \Omega_{\mathbb{P}_{\bullet}}^{\bullet}\right)_{\mathrm{t}} \rightarrow\left(W \mathcal{O}_{X_{\bullet}, K} \rightarrow W \mathcal{O}_{Y_{\bullet}, K}\right)
$$

Taking cohomology, we obtain homomorphisms

$$
b_{U_{\bullet}}^{q}: H_{\text {rig, }, \mathrm{c}}^{q}\left(U_{\bullet} / K\right)^{<1} \rightarrow H_{\mathrm{c}}^{q}\left(U_{\bullet}, W \mathcal{O}_{U_{\bullet}, K}\right),
$$

and a morphism of spectral sequences from (5.3) to (5.4). On the $E_{1}^{i, j}$ terms, this morphism is given by the homomorphisms $b_{U_{i}}^{j}$.

Since the truncation $\operatorname{sk}_{N}^{X}\left(X_{\bullet}\right)$ is an $N$-truncated resolution of $X, X_{n}$ is a projective and smooth $k$-scheme for all $n \leqslant N$. Then the theorem holds for the open subset $U_{n} \subset X_{n}$, which has dimension $d$ since it is étale over $U$. Therefore, the homomorphisms $b_{U_{i}}^{j}$ between the $E_{1}^{i, j}$ terms of (5.3) and (5.4) are isomorphisms for $i \leqslant N$. It follows that $b_{U}^{q}$ is an isomorphism for $q \leqslant N$. 


\section{WitT VECTOR COHOMOLOGY OF SINGULAR VARIETIES}

As the augmentation $\mathbb{P} . \rightarrow \mathbb{P}$ is compatible with the Frobenius liftings, we obtain the following commutative square for all $q$.

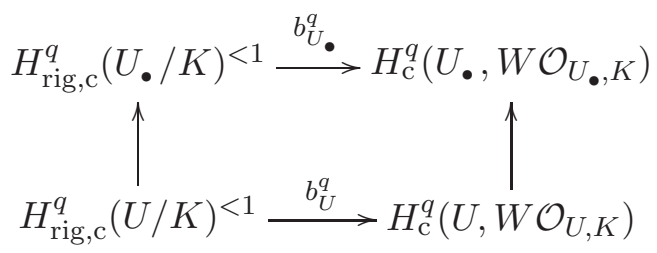

The augmented simplicial schemes $X_{\bullet} \rightarrow X$ and $Y_{\bullet} \rightarrow Y$ are proper hypercoverings, and condition (iv) of Lemma 5.1 implies that $\left(X_{\bullet}, X_{\bullet}, \mathbb{P}_{\bullet}\right)$ and $\left(Y_{\bullet}, Y_{\bullet}, \mathbb{P}_{\bullet}\right)$ are Pr-Pr-Sm-hypercoverings of $(X, X, \mathbb{P})$ and $(Y, Y, \mathbb{P})$ in the sense of [Tsu04, Example 5.1.5]. Therefore, it follows from Tsuzuki's proper descent theorem [Tsu03, Theorem 2.1.3] and [Tsu04, Theorem 5.3.1] that the left vertical arrow is an isomorphism. On the other hand, the right vertical arrow is an isomorphism thanks to Proposition 3.2. Since the source and target of $b_{U}^{q}$ are 0 for $q>2 d$, and $b_{U}^{q}$ is an isomorphism for $q \leqslant 2 d$, this completes the proof of Theorem 1.1 .

\section{Applications and examples}

In this section, we assume that $k$ is a finite field with $q=p^{a}$ elements, and we give applications of Theorem 1.1 to congruences mod $q$ for the number of rational points of some algebraic varieties.

If $X$ is a separated $k$-scheme of finite type, we set for all $i$

$$
P_{i}(X, t)=\operatorname{det}\left(1-t \phi \mid H_{\text {rig,c }}^{i}(X / K)\right),
$$

where $\phi=F^{a}$ is the $k$-linear Frobenius endomorphism of $X$. We recall that, thanks to the Lefschetz trace formula [EL93], the zeta function of $X$ can be expressed as

$$
\zeta(X, t)=\prod_{i} P_{i}(X, t)^{(-1)^{i+1}} .
$$

6.1 We normalize the valuation on $K$ by setting $v_{q}(q)=1$. If $P(t)=\sum_{i} a_{i} t^{i} \in K[t]$, its Newton polygon is the graph of the greatest convex function $s$ on $[0, \operatorname{deg}(P)]$ such that $s(i) \leqslant v_{q}\left(a_{i}\right)$ for all $i$. If $P$ is irreducible, then its Newton polygon is a segment. For any polynomial $P(t) \in 1+t K[t]$, and any $\lambda \in \mathbb{Q}$, we denote by $P^{\lambda}(t)$ the product of the irreducible factors of $P$ whose Newton polygon is a segment of slope $\lambda$, normalized by $P^{\lambda}(0)=1$. For any $\rho \in \mathbb{R}$, we set $P^{<\rho}(t)=\prod_{\lambda<\rho} P^{\lambda}(t)$.

This definition extends by multiplicativity to rational fractions $R(t) \in K(t)$ such that $R(0)=1$. In particular, it can be applied to $\zeta(X, t) \in \mathbb{Q}(t) \subset K(t)$, for any separated $k$-scheme of finite type $X$, and this defines the slope $<\rho$ factor $\zeta^{<\rho}(X, t)$ of $\zeta(X, t)$. By Manin's theorem relating over finite fields the slopes with the eigenvalues of $\phi$, we obtain

$$
\operatorname{det}\left(1-t \phi \mid H_{\text {rig,c }}^{i}(X / K)^{<\rho}\right)=\operatorname{det}\left(1-t \phi \mid H_{\text {rig,c }}^{i}(X / K)\right)^{<\rho} .
$$

On the other hand, we define

$$
\begin{aligned}
P_{i}^{W}(X, t) & =\operatorname{det}\left(1-t \phi \mid H_{\mathrm{c}}^{i}\left(X, W \mathcal{O}_{X, K}\right)\right), \\
\zeta^{W}(X, t) & =\prod_{i} P_{i}^{W}(X, t)^{(-1)^{i+1}} .
\end{aligned}
$$

Then Theorem 1.1 implies Corollary 1.3:

$$
\begin{aligned}
P_{i}^{<1}(X, t) & =P_{i}^{W}(X, t), \quad \text { for all } i, \\
\zeta^{<1}(X, t) & =\zeta^{W}(X, t) .
\end{aligned}
$$

We observe that these polynomials actually have coefficients in $\mathbb{Z}_{p}$. 


\section{P. Berthelot, S. Bloch and H. Esnault}

Proposition 6.2. For any separated $k$-scheme of finite type $X$, and any $\lambda \in \mathbb{Q}$, the polynomials $P_{i}^{\lambda}(X, t)$ and $P_{i}^{W}(X, t)$ belong to $\mathbb{Z}_{p}[t]$.

Proof. By construction, the polynomials $P_{i}(X, t)$ belong to $K[t]$. Let $\phi^{\prime}: X^{\prime} \rightarrow X^{\prime}$ be the pull-back of $\phi$ by the absolute Frobenius endomorphism of $\operatorname{Spec}(k)$. By base change, we obtain

$$
\operatorname{det}\left(1-t \phi^{\prime} \mid H_{\text {rig, }, \mathrm{c}}^{i}\left(X^{\prime} / K\right)\right)=\sigma^{*}\left(P_{i}(X, t)\right) .
$$

On the other hand, the relative Frobenius $F_{X / k}: X \rightarrow X^{\prime}$ commutes with $\phi$ and $\phi^{\prime}$. As it induces an isomorphism $H_{\text {rig,c }}^{i}\left(X^{\prime} / K\right) \stackrel{\sim}{\longrightarrow} H_{\text {rig, }, \mathrm{c}}^{i}(X / K)$, it follows that

$$
\operatorname{det}\left(1-t \phi^{\prime} \mid H_{\text {rig,c }}^{i}\left(X^{\prime} / K\right)\right)=P_{i}(X, t) \text {. }
$$

Thus, $P_{i}(X, t)$ is invariant under $\sigma$, hence belongs to $\mathbb{Q}_{p}[t]$. Moreover, since the Newton polygon of a polynomial does not change by enlarging the field containing its coefficients, the decomposition $P_{i}(X, t)=\prod_{\lambda} P_{i}^{\lambda}(X, t)$ is defined in $\mathbb{Q}_{p}[t]$.

Finally, the slopes of the Frobenius action on $H_{\text {rig }, \mathrm{c}}^{i}(X / K)$ are non-negative [CL99, Theorem 3.1.2], and this is equivalent by Manin's theorem to the fact that the inverses of the roots of $P_{i}(X, t)$ are $p$-adic integers. Therefore, each $P_{i}^{\lambda}(X, t)$ belongs to $\mathbb{Z}_{p}[t]$.

By (6.2), the statement for $P_{i}^{W}(X, t)$ follows (it can also be proved directly by the same argument).

We recall the following well-known result [Ax64], which links congruences modulo some power of $q$ to the triviality of the corresponding slope factor.

Proposition 6.3. Let $\left(N_{r}\right)_{r \geqslant 1}$ be a sequence of integers such that

$$
\Phi(t)=\exp \left(\sum_{r \geqslant 1} N_{r} \frac{t^{r}}{r}\right) \in 1+\mathbb{Z}[[t]],
$$

and assume that $\Phi(t)$ is a rational function in $\mathbb{Q}(t)$. Then $\Phi(t)$ can be written

$$
\Phi(t)=\frac{\prod_{i}\left(1-\alpha_{i} t\right)}{\prod_{j}\left(1-\beta_{j} t\right)},
$$

where $\alpha_{i}, \beta_{j} \in \overline{\mathbb{Z}}$ are such that $\alpha_{i} \neq \beta_{j}$ for all $(i, j)$, and, for any integer $\kappa \geqslant 1$, the following conditions are equivalent:

(i) for all $r \geqslant 1, N_{r} \equiv 0 \bmod q^{\kappa r}$;

(ii) for all $i$ and all $j, \alpha_{i}$ and $\beta_{j}$ are divisible by $q^{\kappa}$ in $\overline{\mathbb{Z}}$;

(iii) for all embeddings $\iota$ of $\overline{\mathbb{Q}}$ in $\overline{\mathbb{Q}}_{p}$, all $i$ and all $j, v_{q}\left(\iota\left(\alpha_{i}\right)\right) \geqslant \kappa, v_{q}\left(\iota\left(\beta_{j}\right)\right) \geqslant \kappa$;

(iv) $\Phi^{<\kappa}(t)=1$.

As explained in the introduction, our first application of Theorem 1.1 over finite fields will be a proof of a consequence of Serre's conjecture on theta divisors.

Proposition 6.4. Let $X$ be a smooth projective variety of pure dimension $n$ over a finite field. Let $D \subset X$ be an ample divisor, of complement $U=X \backslash D$, and assume that the following conditions hold:

(a) the injection $H^{0}\left(X, \omega_{X}\right) \rightarrow H^{0}\left(X, \omega_{X}(D)\right)$ is an isomorphism;

(b) for all $i \geqslant 1$, the canonical homomorphism

$$
H^{n}\left(X, \mathcal{O}_{X}\right) \stackrel{V^{i}}{\longrightarrow} H^{n}\left(X, W_{i+1} \mathcal{O}_{X}\right)
$$

is injective. 


\section{WiTT VECTOR COHOMOLOGY OF SINGULAR VARIETIES}

Then $H_{\text {rig }, \mathrm{c}}^{n}(U / K)^{<1} \rightarrow H_{\text {rig }}^{n}(X / K)^{<1}$ is an isomorphism, and

$$
\zeta^{<1}(X, t)=\zeta^{<1}(D, t) \cdot P_{n}^{<1}(X, t)^{(-1)^{n+1}} .
$$

Proof. Let $\mathcal{I} \cong \mathcal{O}_{X}(-D)$ be the ideal of $D$ in $X$. Condition (a) is equivalent by Serre duality to the condition

$$
H^{n}(X, \mathcal{I}) \stackrel{\sim}{\longrightarrow} H^{n}\left(X, \mathcal{O}_{X}\right) .
$$

Thanks to condition (b), the rows of the commutative diagram

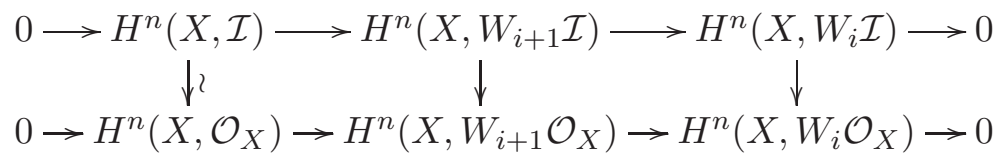

are exact, hence the homomorphism $H^{n}\left(X, W_{i} \mathcal{I}\right) \rightarrow H^{n}\left(X, W_{i} \mathcal{O}_{X}\right)$ is an isomorphism for all $i$. Taking inverse limits and tensoring with $K$, we obtain the isomorphism

$$
H_{\mathrm{c}}^{n}\left(U, W \mathcal{O}_{U, K}\right) \stackrel{\sim}{\longrightarrow} H^{n}\left(X, W \mathcal{O}_{X, K}\right) .
$$

Thus, Theorem 1.1 implies that $H_{\text {rig,c }}^{n}(U / K)^{<1} \rightarrow H_{\text {rig }}^{n}(X / K)^{<1}$ is also an isomorphism.

The multiplicativity of the zeta function shows that it suffices to prove that

$$
\zeta^{<1}(U, t)=P_{n}^{<1}(X, t)^{(-1)^{n+1}} .
$$

By (6.2), we have

$$
P_{i}^{<1}(U, t)=P_{i}^{W}(U, t)
$$

for all $i$. By (6.4), we have $P_{n}^{W}(U, t)=P_{n}^{W}(X, t)=P_{n}^{<1}(X, t)$. On the other hand, $U$ is affine and smooth of pure dimension $n$, hence $H_{\mathrm{c}}^{i}\left(U, W \mathcal{O}_{U, K}\right)=0$ for $i \neq n$ by Corollary 1.2. The proposition follows.

6.5 Proof of Theorem 1.4. Applying Proposition 6.3, it suffices to prove that, if $\Theta$ and $\Theta^{\prime}$ are two theta divisors in an abelian variety $A$, then $\zeta^{<1}(\Theta, t)=\zeta^{<1}\left(\Theta^{\prime}, t\right)$. This will follow from (6.3) if we check that conditions (a) and (b) of Proposition 6.4 hold for a theta divisor in an abelian variety. Since $A$ is an abelian variety, the dualizing bundle $\omega_{A}$ is trivial and, for a theta divisor $\Theta$, the homomorphism $H^{0}\left(A, \mathcal{O}_{A}\right) \rightarrow H^{0}\left(A, \mathcal{O}_{A}(\Theta)\right)$ is an isomorphism [Mum70, III, $\left.\S 16\right]$. Thus, condition (a) is satisfied. As for condition (b), the injectivity of the homomorphisms $H^{n}\left(A, \mathcal{O}_{A}\right) \rightarrow$ $H^{n}\left(A, W_{i+1} \mathcal{O}_{A}\right)$ follows from Serre's theorem on the vanishing of Bockstein operations for abelian varieties in characteristic $p$ (see [Ser58a, 1, §3] and [Ser58b, Théorème 2]).

Remarks 6.6. (i) In fact, Serre's conjecture is phrased more motivically: the difference of the motives of $\Theta$ and $\Theta^{\prime}$ should be divisible in a suitable sense by the Lefschetz motive. What we show in Theorem 1.4 is a finite field implication of the motivic assertion. The motivic statement is clearly stronger than this implication. On the other hand, it is hard to approach directly as it deals with non-effective motives.

(ii) The property of the theorem is very special for theta divisors. It is of course not true in general that two effective divisors $D, D^{\prime}$ with $h^{0}(\mathcal{O}(D))=h^{0}\left(\mathcal{O}\left(D^{\prime}\right)\right)$ carry the same number of points modulo $q$ : take for example $D=2\{0\}, D^{\prime}=\{0\}+\{\infty\}$ on $\mathbb{P}^{1}$.

(iii) Let us also remark that over the complex numbers, when $\Theta$ is irreducible, one knows that its singularities are rational [EL97, Theorem 3.3]. A precise analogy of this over a finite field is not quite clear, as the notion of rational singularities itself requires the resolution of singularities. However, it should be related to the assertion of Corollary 1.3.

According to Grothendieck-Deligne's philosophy of motives, Hodge type over the field of complex numbers behaves the same way as congruences for the number of rational points. In view 


\section{P. Berthelot, S. Bloch and H. Esnault}

of the previous remarks, it is worth pointing out that Theorem 1.4 has the following Hodge theoretic analogue (where $H^{*}(X)$ denotes the classical complex cohomology of an algebraic scheme $X$ over $\mathbb{C})$.

Proposition 6.7. Let $A$ be an abelian variety of dimension $n$ over $\mathbb{C}$, and $\Theta \subset A$ a theta divisor. Let $F$ be the Deligne's Hodge filtration on $H^{i}(A)$ and $H^{i}(\Theta)$. Then the restriction map $\operatorname{gr}_{F}^{0} H^{i}(A) \rightarrow$ $\operatorname{gr}_{F}^{0} H^{i}(\Theta)$ is an isomorphism for $i \neq n$. In particular, if $\Theta^{\prime}$ is another theta divisor, $\operatorname{dim} \operatorname{gr}_{F}^{0} H^{i}(\Theta)=$ $\operatorname{dim} \operatorname{gr}_{F}^{0} H^{i}\left(\Theta^{\prime}\right)$ for all $i$.

Proof. This is a simple example of the application of [Esn90, Proposition 1.2]. Since $\Theta$ is ample, we know by the weak Lefschetz theorem that the restriction map $H^{i}(A) \rightarrow H^{i}(\Theta)$ is an isomorphism for $i \leqslant n-2$, and is injective for $i=n-1$. Also $\operatorname{gr}_{F}^{0} H^{i}(X)=0$ for $i>\operatorname{dim}(X)$ for any separated $\mathbb{C}$-scheme of finite type $X$ (see [Del74, Théorème 8.2.4]). Thus, all we have to prove is

$$
\operatorname{gr}_{F}^{0} H_{c}^{n}(U) \stackrel{\sim}{\longrightarrow} \operatorname{gr}_{F}^{0} H^{n}(A),
$$

with $U=A \backslash \Theta$. Let $\sigma: A^{\prime} \rightarrow A$ be a birational morphism such that $\left.\sigma\right|_{U}$ is an isomorphism, $A^{\prime}$ is smooth, and $D=\sigma^{-1}(\Theta)_{\text {red }}$ is a normal crossings divisor. Then one has $\operatorname{gr}_{F}^{0} H_{c}^{n}(U)=$ $H^{n}\left(A^{\prime}, \mathcal{O}_{A^{\prime}}(-D)\right)$, and $\sigma^{*}: \operatorname{gr}_{F}^{0} H^{n}(A)=H^{n}\left(A, \mathcal{O}_{A}\right) \rightarrow \operatorname{gr}_{F}^{0} H^{n}\left(A^{\prime}\right)=H^{n}\left(A^{\prime}, \mathcal{O}_{A^{\prime}}\right)$ is an isomorphism. One the other hand, the composed morphism

$$
H^{n}\left(A, \mathcal{O}_{A}(-\Theta)\right) \stackrel{\sigma^{*}}{\longrightarrow} H^{n}\left(A^{\prime}, \mathcal{O}_{A^{\prime}}(-D)\right) \stackrel{\iota}{\rightarrow} H^{n}\left(A^{\prime}, \mathcal{O}_{A^{\prime}}\right) \stackrel{\sim}{\longleftarrow} H^{n}\left(A, \mathcal{O}_{A}\right)
$$

is an isomorphism since $\Theta$ is a theta divisor, while $\sigma^{*}, \iota$ are surjective for dimension reasons. Thus, all maps in (6.6) are isomorphisms, which in particular proves the proposition.

We now consider the case of intersections of hypersurfaces of small degrees.

Proposition 6.8. Let $k$ be a perfect field of characteristic $p$, let $K=\operatorname{Frac}(W(k))$, and let $D_{1}, \ldots, D_{r} \subset \mathbb{P}_{k}^{n}$ be hypersurfaces of degrees $d_{1}, \ldots, d_{r}$. Assume that $\sum_{j} d_{j} \leqslant n$. Then, if $Z=$ $D_{1} \cap \cdots \cap D_{r}$,

$$
H^{0}\left(Z, W \mathcal{O}_{Z, K}\right)=K, \quad H^{i}\left(Z, W \mathcal{O}_{Z, K}\right)=0 \quad \text { for } i \geqslant 1 .
$$

Proof. We proceed by induction on $r$. Let $r=1, D=D_{1}=Z, d=d_{1}$. Then the condition $d \leqslant n$ implies that the homomorphisms $H^{i}\left(\mathbb{P}^{n}, \mathcal{O}_{\mathbb{P}^{n}}\right) \rightarrow H^{i}\left(D, \mathcal{O}_{D}\right)$ are isomorphisms for all $i$. It follows that, for any $m \geqslant 1$,

$$
H^{0}\left(D, W_{m} \mathcal{O}_{D}\right)=W_{m}(k), \quad H^{i}\left(D, W_{m} \mathcal{O}_{D}\right)=0 \quad \text { for } i \geqslant 1,
$$

which implies (6.7).

For arbitrary $r$, let $D=D_{1} \cup \cdots \cup D_{r}$, which is a hypersurface of degree $d=\sum_{j} d_{j} \leqslant n$. For each sequence $1 \leqslant i_{0}<\cdots<i_{s} \leqslant r$, let $Z_{i_{0}, \ldots, i_{s}}=D_{i_{0}} \cap \cdots \cap D_{i_{s}}$. By Corollary 2.3, we obtain an exact sequence

$$
0 \rightarrow W \mathcal{O}_{D, K} \rightarrow \prod_{i=1}^{r} W \mathcal{O}_{Z_{i}, K} \rightarrow \cdots \rightarrow \prod_{i=1}^{r} W \mathcal{O}_{Z_{1, \ldots, \hat{i}, \ldots, r}, K} \rightarrow W \mathcal{O}_{Z, K} \rightarrow 0 .
$$

Applying the previous result to $D$, and the induction hypothesis to all $Z_{i_{0}, \ldots, i_{j}}$ for $j \leqslant r-2$, we can view this exact sequence as providing a $\Gamma\left(\mathbb{P}^{n},-\right)$-acyclic left resolution of $W \mathcal{O}_{Z, K}$. Taking sections and observing that the complex

$$
0 \rightarrow K \rightarrow \prod_{i=1}^{r} K \rightarrow \cdots \rightarrow \prod_{i=1}^{r} K \rightarrow 0
$$

is acyclic, except in degree 0 where its cohomology is equal to $K$, we obtain (6.7). 


\section{WiTT VECTOR COHOMOLOGY OF SINGULAR VARIETIES}

Proof of Corollary 1.5. Combining Proposition 6.8 with Corollary 1.3, we obtain

$$
\zeta^{<1}(Z, t)=\frac{1}{1-t}
$$

which, by Proposition 6.3, is equivalent to congruence (1.7) for all finite extensions of $\mathbb{F}_{q}$.

We now discuss some cases where, given a morphism $f: X \rightarrow Y$ between two varieties over a finite field, Theorem 1.1 provides congruences between the numbers of rational points on $X$ and $Y$.

Proposition 6.9. (i) Let $f: X \rightarrow Y$ be a proper morphism between two separated $\mathbb{F}_{q}$-schemes of finite type. If the induced homomorphisms $f^{*}: H_{\mathrm{c}}^{i}\left(Y, W \mathcal{O}_{Y, K}\right) \rightarrow H_{\mathrm{c}}^{i}\left(X, W \mathcal{O}_{X, K}\right)$ are isomorphisms for all $i \geqslant 0$, then

$$
\left|X\left(\mathbb{F}_{q}\right)\right| \equiv\left|Y\left(\mathbb{F}_{q}\right)\right| \bmod q .
$$

(ii) Let $X$ be a proper scheme over $\mathbb{F}_{q}$, and $G$ a finite group acting on $X$ so that each orbit is contained in an affine open subset. If the action of $G$ on $H^{i}\left(X, W \mathcal{O}_{X, K}\right)$ is trivial for all $i$, then

$$
\left|X\left(\mathbb{F}_{q}\right)\right| \equiv\left|(X / G)\left(\mathbb{F}_{q}\right)\right| \bmod q .
$$

Proof. Assertion (i) is an immediate consequence of Corollary 1.3 and Proposition 6.3, and implies assertion (ii) thanks to the following lemma.

Lemma 6.10. Let $X$ be a proper scheme over $\mathbb{F}_{q}, G$ a finite group acting on $X$ so that each orbit is contained in an affine open subset, $f: X \rightarrow Y:=X / G$ the quotient map. Then $f^{*}$ induces canonical isomorphisms

$$
H^{i}\left(Y, W \mathcal{O}_{Y, K}\right) \stackrel{\sim}{\longrightarrow} H^{i}\left(X, W \mathcal{O}_{X, K}\right)^{G}
$$

for all $i$.

The canonical morphism $f: X \rightarrow Y$ is finite, and $\mathcal{O}_{Y} \stackrel{\sim}{\longrightarrow} f_{*}\left(\mathcal{O}_{X}\right)^{G}$. By induction on $m$, $R^{i} f_{*}\left(W_{m} \mathcal{O}_{X}\right)=0$ for all $i \geqslant 1$ and all $m \geqslant 1$. Moreover, the morphisms

$$
W_{m} \mathcal{O}_{Y} \rightarrow W_{m}\left(f_{*}\left(\mathcal{O}_{X}\right)^{G}\right) \rightarrow W_{m}\left(f_{*}\left(\mathcal{O}_{X}\right)\right)^{G} \cong f_{*}\left(W_{m} \mathcal{O}_{X}\right)^{G}
$$

are isomorphisms for all $m \geqslant 1$. Since taking invariants under $G$ commutes with inverse limits and with tensorization by $\mathbb{Q}$, they provide isomorphisms

$$
W \mathcal{O}_{Y, K} \stackrel{\sim}{\longrightarrow}\left(f_{*}\left(W \mathcal{O}_{X}\right)^{G}\right)_{K} \stackrel{\sim}{\longrightarrow} f_{*}\left(W \mathcal{O}_{X, K}\right)^{G} .
$$

As $\operatorname{char}(K)=0$, taking invariants under $G$ commutes with cohomology for $K[G]$-modules, and we can write

$$
H^{i}\left(Y, W \mathcal{O}_{Y, K}\right) \stackrel{\sim}{\longrightarrow} H^{i}\left(Y, f_{*}\left(W \mathcal{O}_{X, K}\right)^{G}\right) \stackrel{\sim}{\rightarrow} H^{i}\left(Y, f_{*}\left(W \mathcal{O}_{X, K}\right)\right)^{G} .
$$

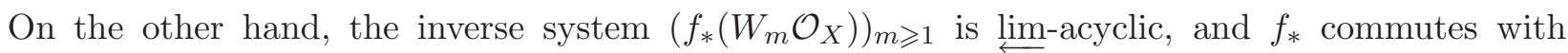
tensorization with $K$. Therefore, the morphism

$$
f_{*}\left(W \mathcal{O}_{X, K}\right) \rightarrow \mathbb{R} f_{*}\left(W \mathcal{O}_{X, K}\right)
$$

is an isomorphism, and we obtain

$$
H^{i}\left(Y, f_{*}\left(W \mathcal{O}_{X, K}\right)\right)^{G \stackrel{\sim}{\longrightarrow}} H^{i}\left(X, W \mathcal{O}_{X, K}\right)^{G},
$$

which proves the lemma.

6.11 In the most favorable cases, the assumptions of the previous proposition can be checked in characteristic $p$. This is the case under the assumptions of Corollary 1.6, the proof of which follows. 


\section{P. Berthelot, S. Bloch and H. Esnault}

Proof of Corollary 1.6. Let $f: X \rightarrow Y$ be a morphism between two proper $\mathbb{F}_{q^{-}}$-schemes such that the induced homomorphisms $f^{*}: H^{i}\left(Y, \mathcal{O}_{Y}\right) \rightarrow H^{i}\left(X, \mathcal{O}_{X}\right)$ are isomorphisms for all $i \geqslant 0$. By induction on $m$, it follows that $H^{i}\left(Y, W_{m} \mathcal{O}_{Y}\right) \rightarrow H^{i}\left(X, W_{m} \mathcal{O}_{X}\right)$ is an isomorphism for all $i \geqslant 0$ and all $m \geqslant 1$. As $X$ and $Y$ are proper, we obtain that

$$
H^{i}\left(Y, W \mathcal{O}_{Y, K}\right) \stackrel{\sim}{\longrightarrow} H^{i}\left(X, W \mathcal{O}_{X, K}\right)
$$

for all $i \geqslant 0$. Then congruence (1.8) again follows from Corollary 1.3 and Proposition 6.3.

Corollary 6.12. Let $X$ be a proper scheme over $\mathbb{F}_{q}$, and $G$ a finite group acting on $X$ so that each orbit is contained in an affine open subset of $X$. If $|G|$ is prime to $p$, and if the action of $G$ on $H^{i}\left(X, \mathcal{O}_{X}\right)$ is trivial for all $i$, then

$$
\left|X\left(\mathbb{F}_{q}\right)\right| \equiv\left|(X / G)\left(\mathbb{F}_{q}\right)\right| \bmod q .
$$

Proof. When $|G|$ is prime to $p$, taking invariants under $G$ is an exact functor on $k[G]$-modules, and we obtain isomorphisms

$$
\begin{aligned}
H^{i}\left(Y, \mathcal{O}_{Y}\right) \stackrel{\sim}{\longrightarrow} H^{i}\left(Y,\left(\mathbb{R} f_{*} \mathcal{O}_{X}\right)^{G}\right) & \stackrel{\sim}{\longrightarrow} H^{i}\left(Y,\left(\mathbb{R} f_{*} \mathcal{O}_{X}\right)\right)^{G} \\
& \stackrel{\sim}{\longrightarrow} H^{i}\left(X, \mathcal{O}_{X}\right)^{G}=H^{i}\left(X, \mathcal{O}_{X}\right) .
\end{aligned}
$$

Hence, we can apply Corollary 1.6.

Remarks 6.13. Without assumption on $|G|, \mathrm{Fu}$ and Wan have proved [FW05, Theorem 0.1] that the congruence (6.9) holds under the following hypotheses:

(a) $X$ is the reduction of a projective and smooth $W$-scheme $X^{\prime}$ with $p$-torsion free Hodge cohomology;

(b) the action of $G$ on $X$ is the reduction of an action on $X^{\prime}$ such that the induced action on $H^{i}\left(X^{\prime}, \mathcal{O}_{X^{\prime}}\right)$ is trivial for all $i$.

We do not know whether, under these hypotheses, the action of $G$ is trivial on the spaces $H^{i}\left(X, W \mathcal{O}_{X, K}\right)$. However, the following example seems to indicate that, when $p$ divides $|G|$, a mod $p$ assumption on the action of $G$ as in Corollary 6.12 does not suffice to provide congruences such as $(6.9)$.

Let $p=2$, let $E_{1}$ be an elliptic curve over $\mathbb{F}_{q}$ with an $\mathbb{F}_{q}$-rational point $t$ of order 2 , and let $E_{2}$ be another elliptic curve over $\mathbb{F}_{q}$. We define $X=E_{1} \times E_{2}$, and we let the group $G=\mathbb{Z} / 2 \mathbb{Z}$ act on $X$ via $(x, y) \mapsto(x+t,-y)$, so that $Y=X / G$ is the classical Igusa surface [Igu55], which is smooth over $\mathbb{F}_{q}$. The action of $G$ on $H^{i}\left(X, \mathcal{O}_{X}\right)$ is trivial for all $i$, but, thanks to the Künneth formula in crystalline cohomology, one checks easily that $H^{2}\left(X, W \mathcal{O}_{X, K}\right) \cong H^{2}(X / K)^{<1} \neq 0$, while $H^{2}\left(Y, W \mathcal{O}_{Y, K}\right) \cong\left(H^{2}(X / K)^{G}\right)^{<1}=0$. Therefore, $\zeta^{<1}(Y, t) \neq \zeta^{<1}(X, t)$, and a congruence such as (6.9) cannot hold for all powers of $q$.

Note also that, for any $p$, taking the quotient of an abelian variety by the subgroup generated by a rational point of order $p$ provides an example where the assumptions of Proposition 6.9(ii) are satisfied, while those of Corollary 1.6 fail to be true.

\section{ACKNOWLEDGEMENTS}

It is a pleasure to thank Jean-Pierre Serre for his strong encouragement and his help. Theorem 1.4 was the main motivation for this work. We thank Luc Illusie for useful discussions. The third named author thanks Eckart Viehweg for his interest and encouragement. Corollary 1.2 was inspired by the analogy on the relation between de Rham and coherent cohomology, which has been developed jointly with him. 


\section{WiTT VECTOR COHOMOLOGY OF SINGULAR VARIETIES}

\section{REFERENCES}

Ax64 J. Ax, Zeroes of polynomials over finite fields, Amer. J. Math. 86 (1964), 255-261.

Ber86 P. Berthelot, Géométrie rigide et cohomologie des variétés algébriques de caractéristique p, Journées d'Analyse p-adique, Luminy, 1982, in Introduction aux cohomologies p-adiques, Mém. Soc. Math. France (N.S.) 23 (1986), 7-32.

Ber96 P. Berthelot, Cohomologie rigide et cohomologie rigide à supports propres, première partie, Preprint IRMAR 96-03, Université de Rennes (1996).

Ber97a P. Berthelot, Finitude et pureté cohomologique en cohomologie rigide, with an appendix by A. J. de Jong, Invent. Math. 128 (1997), 329-377.

Ber97b P. Berthelot, Dualité de Poincaré et formule de Künneth en cohomologie rigide, C. R. Acad. Sci. Paris Sér. I Math. 325 (1997), 493-498.

Blo77 S. Bloch, Algebraic K-theory and crystalline cohomology, Publ. Math. Inst. Hautes Études Sci. 47 (1977), 187-268.

BEL05 S. Bloch, H. Esnault and M. Levine, Decomposition of the diagonal and eigenvalues of Frobenius for Fano hypersurfaces, Amer. J. Math. 127 (2005), 193-207.

BH69 T. Bloom and M. Herrera, De Rham cohomology of an analytic space, Invent. Math. 7 (1969), 275-296.

CL99 B. Chiarellotto and B. Le Stum, Pentes en cohomologie rigide et F-isocristaux unipotents, Manuscripta Math. 100 (1999), 455-468.

CT03 B. Chiarellotto and N. Tsuzuki, Cohomological descent of rigid cohomology for étale coverings, Rend. Sem. Mat. Univ. Padova 109 (2003), 63-215.

Dej96 J. de Jong, Smoothness, semi-stability and alterations, Publ. Math. Inst. Hautes Études Sci. 83 (1996), 51-93.

Del74 P. Deligne, Théorie de Hodge III, Publ. Math. Inst. Hautes Études Sci. 44 (1974), 5-77.

EL97 L. Ein and R. Lazarsfeld, Singularities of theta divisors and the birational geometry of irregular varieties, J. Amer. Math. Soc. 10 (1997), 243-258.

Esn90 H. Esnault, Hodge type of subvarieties of $\mathbb{P}^{n}$ of small degrees, Math. Ann. 288 (1990), 549-551.

Esn05 H. Esnault, Appendix to "Congruences for rational points on varieties defined over finite fields" by N. Fakhruddin and C. S. Rajan, Math. Ann. 333 (2005), 811-814.

EL93 J.-Y. Étesse and B. Le Stum, Fonctions L associées aux F-isocristaux surconvergents. I, Math. Ann. 296 (1993), 557-576.

FW05 L. Fu and D. Wan, Mirror congruences for rational points on Calabi-Yau varieties, Asian J. Math. 10 (2006), 1-10.

Gro61 A. Grothendieck, Éléments de géométrie algébrique, Publ. Math. Inst. Hautes Études Sci. 11 (1961), $5-167$.

Har66 R. Hartshorne, Residues and duality, Lecture Notes in Mathematics, vol. 20 (Springer, Berlin, 1966).

Igu55 J.-I. Igusa, On some problems in abstract algebraic geometry, Proc. Natl. Acad. Sci. USA 41 (1955), 964-967.

Ill79 L. Illusie, Complexe de de Rham-Witt et cohomologie cristalline, Ann. Sci. École. Norm. Sup. (4) 12 (1979), 501-661.

Kat71 N. Katz, On a theorem of Ax, Amer. J. Math. 93 (1971), 485-499.

Kie67 R. Kiehl, Theorem A und Theorem B in der nichtarchimedischen Funktionentheorie, Invent. Math. 2 (1967), 256-273.

Mum70 D. Mumford, Abelian varieties (Tata Institute of Fundamental Research, Tata, 1970).

Ser58a J.-P. Serre, Sur la topologie des variétés algébriques en caractéristique $p$, in Proc. symp. internacional de topología algebraica (Universidad Nacional Autónoma de México and UNESCO, Mexico City, 1958), 24-53.

Ser58b J.-P. Serre, Quelques propriétés des variétés abéliennes en caractéristique p, Amer. J. Math. 80 (1958), 715-739. 


\section{WitT VECTOR COHOMOLOGY OF SINGULAR VARIETIES}

Ser62 J.-P. Serre, Corps locaux, Publications de l'Institut de Mathématique de l'Université de Nancago, vol. VIII (Hermann, Paris, 1962).

Tsu03 N. Tsuzuki, Cohomological descent of rigid cohomology for proper coverings, Invent. Math. 151 (2003), 101-133.

Tsu04 N. Tsuzuki, Cohomological descent in rigid cohomology, in Geometric aspects of Dwork theory, vol. 2, eds A. Adolphson et al. (de Gruyter, Berlin, 2004), 931-981.

Wan04 D. Wan, Mirror symmetry for zeta functions, Preprint (2004), in Mirror Symmetry V, AMS/IP Studies in Advanced Mathematics, vol. 38 (American Mathematical Society, Providence, RI, 2007), to appear.

Pierre Berthelot pierre.berthelot@univ-rennes1.fr

IRMAR, Université de Rennes 1, Campus de Beaulieu, 35042 Rennes cedex, France

Spencer Bloch bloch@math.uchicago.edu

Department of Mathematics, University of Chicago, Chicago, IL 60637, USA

Hélène Esnault esnault@uni-essen.de

Mathematik, Universität Duisburg-Essen, FB6, Mathematik, 45117 Essen, Germany 